\title{
Heat kernel estimates for general symmetric pure jump Dirichlet forms
}

\author{
Zhen-Qing Chen, Takashi Kumagai and Jian Wang
}

\begin{abstract}
In this paper, we consider the following symmetric non-local Dirichlet forms of pure jump type on metric measure space $(M, d, \mu)$ :

$$
\mathcal{E}(f, g)=\int_{M \times M}(f(x)-f(y))(g(x)-g(y)) J(d x, d y),
$$

where $J(d x, d y)$ is a symmetric Radon measure on $M \times M \backslash$ diag that may have different scalings for small jumps and large jumps. Under general volume doubling condition on $(M, d, \mu)$ and some mild quantitative assumptions on $J(d x, d y)$ that are allowed to have light tails of polynomial decay at infinity, we establish stability results for two-sided heat kernel estimates as well as heat kernel upper bound estimates in terms of jumping kernel bounds, the cut-off Sobolev inequalities, and the Faber-Krahn inequalities (resp. the Poincaré inequalities). We also give stable characterizations of the corresponding parabolic Harnack inequalities.
\end{abstract}

AMS 2010 Mathematics Subject Classification: Primary 60J35, 35K08, 60J75; Secondary 31C25, 60J25, 60J45.

Keywords and phrases: symmetric non-local Dirichlet form, symmetric jump process, metric measure space, heat kernel estimate, jumping kernel, cut-off Sobolev inequality, Faber-Krahn inequality, Poincaré inequality, parabolic Harnack inequality

\section{Contents}

1 Introduction and main results 2

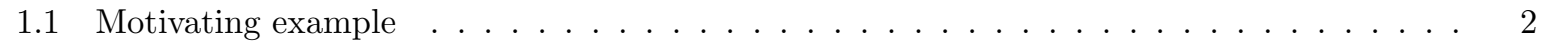

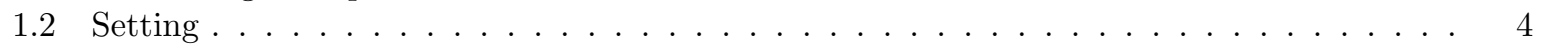

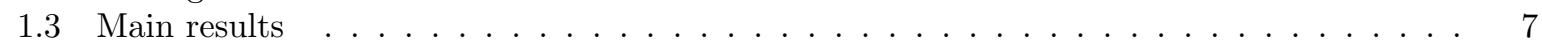

1.4 Remarks and discussions . . . . . . . . . . . . . . . . . . . 11

2 Implications of heat kernel estimates

$2.1 \operatorname{UHK}\left(\phi_{j}, \phi_{c}\right)+(\mathcal{E}, \mathcal{F})$ is conservative $\Longrightarrow \mathrm{J}_{\phi_{j}, \leq}$ and $\mathrm{HK}_{-}\left(\phi_{j}, \phi_{c}\right) \Longrightarrow \mathrm{J}_{\phi_{j}} \ldots \ldots \ldots$

$2.2 \operatorname{UHK}\left(\phi_{j}, \phi_{c}\right)+(\mathcal{E}, \mathcal{F})$ is conservative $\Longrightarrow \operatorname{Gcap}(\phi)+\operatorname{CSJ}(\phi) \ldots \ldots \ldots \ldots \ldots$

3 Implications of $\mathrm{FK}(\phi), \operatorname{CSJ}(\phi)$ and $\mathrm{J}_{\phi_{j}, \leq}\left(\right.$ or $\left.\mathrm{J}_{\phi, \leq}\right)$

$3.1 \mathrm{FK}(\phi)+\mathrm{J}_{\phi, \leq}+\operatorname{CSJ}(\phi) \Rightarrow \mathrm{E}_{\phi}$, and $\mathrm{FK}(\phi)+\mathrm{J}_{\phi, \leq}+\mathrm{E}_{\phi} \Rightarrow \operatorname{UHKD}(\phi) \ldots \ldots \ldots$

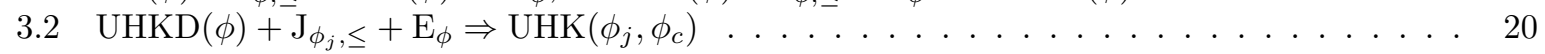

4 Characterizations of $\mathrm{HK}_{-}\left(\phi_{j}, \phi_{c}\right)$ and $\operatorname{HK}\left(\phi_{j}, \phi_{c}\right) \quad \mathbf{2 9}$

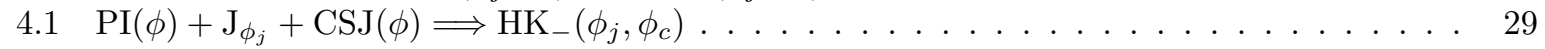

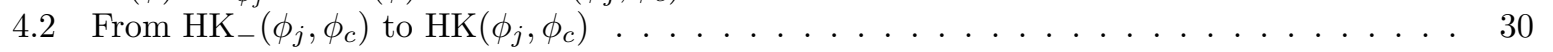


5 Applications $\mathbf{3 0}$

5.1 Parabolic Harnack inequalities . . . . . . . . . . . . . . . . . . . . . . 30

5.2 Transferring method for heat kernel estimates and parabolic Harnack inequalities . . . . . 31

5.3 Examples ................................ . . . . . . . . .

6 Appendix: Proof of assertions in Example 1.1

\section{Introduction and main results}

\section{$1.1 \quad$ Motivating example}

This paper can be regarded as a sequel to [CKW1, CKW2] and [CKW4, where stability of heat kernel estimates and parabolic Harnack inequalities are studied for symmetric pure jump Markov processes and for symmetric diffusions with jumps. In these papers, the scale functions for large jumps are all assumed to be less than that for the diffusion processes if there is one (for example, $r \mapsto r^{2}$ in the Euclidean space case). In this paper, we consider general symmetric pure jump Markov processes whose jumping kernel can have light tails at infinity. New phenomena arise in the light tail case. To illustrate some of the new phenomena, we start with an example.

Example 1.1. Let $(M, d)$ be a locally compact separable metric space, and $\mu$ a positive Radon measure on $M$ with full support. We will refer to such a triple $(M, d, \mu)$ as a metric measure space. Let $V(x, r)=\mu(B(x, r))$ for any $x \in M$ and $r>0$. Assume that the volume doubling (VD) condition holds, i.e., there is a constant $c_{\mu}>0$ so that

$$
V(x, 2 r) \leq c_{\mu} V(x, r), \quad x \in M, r>0 .
$$

Suppose $X:=\left\{X_{t}, t \geq 0 ; \mathbb{P}^{x}, x \in M\right\}$ is a conservative symmetric strong Markov process on $M$ that has a transition density function $q(t, x, y)$ with respect to $\mu$ such that

$$
q(t, x, y) \asymp \frac{1}{V\left(x, t^{1 / \beta}\right)} \exp \left(-c\left(\frac{d(x, y)^{\beta}}{t}\right)^{1 /(1-\beta)}\right), \quad t>0, x, y \in M
$$

for some $\beta \geq 2$. Here and in what follows, for two positive functions $f(t, z)$ and $g(t, z)$, notation $f \asymp g$ means that there exist positive constants $c_{i}(i=1, \cdots, 4)$ such that

$$
c_{1} f\left(c_{2} t, z\right) \leq g(t, z) \leq c_{3} f\left(c_{4} t, z\right) .
$$

Let $S:=\left(S_{t}\right)_{t \geq 0}$ be a subordinator with $S_{0}=0$ that is independent of $X$ and has the Laplace exponent

$$
f(r)=\int_{0}^{\infty}\left(1-e^{-r s}\right) \nu(s) d s, \quad r>0
$$

where

$$
\nu(s)=\frac{1}{s^{1+\gamma_{1}}} \mathbf{1}_{\{0<s \leq 1\}}+\frac{1}{s^{1+\gamma_{2}}} \mathbf{1}_{\{s>1\}}
$$

with $\gamma_{1} \in(0,1)$ and $\gamma_{2} \in(1, \infty)$. Let $Y:=\left(Y_{t}\right)_{t \geq 0}$ be the subordinate process defined by $Y_{t}:=X_{S_{t}}$ for all $t>0$. For a set $A \subset M$, define the exit time $\tau_{A}^{Y}=\inf \left\{t>0: Y_{t} \notin A\right\}$. The subordinate process $Y$ has the following properties.

Claim 1. $Y$ is a symmetric jump process such that 
(i) its jumping kernel $J(d x, d y)$ has a density with respect to the product measure $\mu \times \mu$ on $M \times M \backslash$ diag given by

$$
J(x, y) \simeq \begin{cases}\frac{1}{V(x, d(x, y)) d(x, y)^{\alpha_{1}}}, & d(x, y) \leq 1 \\ \frac{1}{V(x, d(x, y)) d(x, y)^{\alpha_{2}}}, & d(x, y) \geq 1\end{cases}
$$

where $\alpha_{1}=\gamma_{1} \beta$ and $\alpha_{2}=\gamma_{2} \beta$, and diag stands for the diagonal of $M \times M$.

(ii) for any $x_{0} \in M$ and $r>0$,

$$
\mathbb{E}^{x}\left[\tau_{B\left(x_{0}, r\right)}^{Y}\right] \simeq r^{\alpha_{1}} \vee r^{\beta}
$$

and

$$
\operatorname{cap}_{Y}\left(B\left(x_{0}, r\right), B\left(x_{0}, 2 r\right)\right) \simeq \begin{cases}V\left(x_{0}, r\right) / r^{\alpha_{1}} & \text { for } r \leq 1 \\ V\left(x_{0}, r\right) / r^{\beta} & \text { for } r>1\end{cases}
$$

Here, for any subsets $A \subset B$, the relative capacity $\operatorname{cap}_{Y}(A, B)$ is defined as

$$
\operatorname{cap}_{Y}(A, B):=\inf \left\{\mathcal{E}_{Y}(\varphi, \varphi): \varphi=1 \mathcal{E}_{Y} \text {-q.e. on } A \text { and } \varphi=0 \mathcal{E}_{Y} \text {-q.e. on } B^{c}\right\},
$$

where $\mathcal{E}_{Y}(\cdot, \cdot)$ is the non-local Dirichlet form associated with the process $Y$. In this paper, we use $:=$ as a way of definition. We write $f \simeq g$, if there exist constants $c_{1}, c_{2}>0$ such that $c_{1} g(x) \leq f(x) \leq c_{2} g(x)$ for the specified range of $x$. Similarly, we write $f \preceq g$ if there exists a constant $c>0$ such that $f(x) \leq c g(x)$. For real numbers $a$ and $b$, set $a \vee b:=\max \{a, b\}$ and $a \wedge b:=\min \{a, b\}$.

Claim 2. The process $Y$ has a jointly continuous transition density function $p(t, x, y)$ with respect to the measure $\mu$ on $M$ so that

$$
p(t, x, y) \simeq \frac{1}{V\left(x, t^{1 / \alpha_{1}}\right)} \wedge(t J(x, y)) \quad \text { for } t \leq 1
$$

and

$$
\begin{aligned}
& c_{1}\left(\frac{1}{V\left(x, t^{1 / \beta}\right)} \mathbf{1}_{\left\{d(x, y) \leq t^{1 / \beta}\right\}}+\frac{t}{V(x, d(x, y)) d(x, y)^{\alpha_{2}}} \mathbf{1}_{\left\{d(x, y)>t^{1 / \beta}\right\}}\right) \\
& \leq p(t, x, y) \leq c_{2}\left(\frac{1}{V\left(x, t^{1 / \beta}\right)} \wedge\left(t J(x, y)+q\left(c_{3} t, x, y\right)\right)\right) \quad \text { for } t \geq 1
\end{aligned}
$$

where $q(t, x, y)$ is the transition density function for the diffusion process $X$ of the form (1.1). If, in addition, $(M, d, \mu)$ is connected and satisfies the chain condition, then

$$
p(t, x, y) \asymp \begin{cases}\frac{1}{V\left(x, t^{1 / \alpha}\right)} \wedge(t J(x, y)), & t \leq 1, \\ \frac{1}{V\left(x, t^{1 / \beta}\right)} \wedge(t J(x, y)+q(t, x, y)), & t \geq 1 .\end{cases}
$$

The proof of Example 1.1 will be given in the appendix at the end of this paper. The first assertion (i) of Claim 1 says that the scaling function of jumping kernel for the process $Y$ is $\phi_{j}(r):=r^{\alpha_{1}} \vee r^{\alpha_{2}}$ with $\alpha_{1}<\beta<\alpha_{2}$, while the second assertion (ii) of Claim 1 indicates that the scaling function of the process $Y$ is $r^{\alpha_{1}} \vee r^{\beta}$. The scaling function of the process $Y$ is different from the associated jumping kernel at large scale (that is, when $r>1$ ). Thus the behavior of the symmetric jump process $Y$ in Example 1.1 is different from that of symmetric $\alpha_{1}$-stable-like 
or mixed stable-like processes studied in CK, CKW1. This may appear surprising at the first glance that the process $Y$ enjoys the diffusive scaling $\phi_{c}(r):=r^{\beta}$ when $r>1$. But it becomes quite reasonable if one thinks more about it as the jumping kernel $J(d x, d y)$ of $Y$ has finite second moment in the case of $\beta=2$. Note that in this example, $\phi_{j}(r) \geq \phi_{c}(r)$ for all $r>0$.

The goal of this paper is to study heat kernel estimates and their applications systematically for general pure jump Dirichlet forms, which include a large class of symmetric pure jump Dirichlet forms that have light jumping kernel at infinity and thus possibly exhibit diffusive behaviors. The main results of this paper are Theorems 1.11, 1.12 and 1.15 .

\subsection{Setting}

Let $(M, d, \mu)$ be a measure metric space. Throughout the paper, we assume that all balls are relatively compact, and assume for simplicity that $\mu(M)=\infty$. We emphasize that in this paper we do not assume $M$ to be connected nor $(M, d)$ to be geodesic. We are concerned with regular Dirichlet forms $(\mathcal{E}, \mathcal{F})$ on $L^{2}(M ; \mu)$ that only have pure-jump part; namely,

$$
\mathcal{E}(f, g)=\int_{M \times M \backslash \operatorname{diag}}(f(x)-f(y)(g(x)-g(y)) J(d x, d y), \quad f, g \in \mathcal{F},
$$

where $J(\cdot, \cdot)$ is a symmetric Radon measure on $M \times M \backslash$ diag.

Throughout the paper, we will always take a quasi-continuous version of $f \in \mathcal{F}$ without denoting it by $\tilde{f}$. Let $(\mathcal{L}, \mathcal{D}(\mathcal{L}))$ be the (negative definite) $L^{2}$-generator of $(\mathcal{E}, \mathcal{F})$ on $L^{2}(M ; \mu)$, and let $\left\{P_{t}\right\}_{t \geq 0}$ be the associated semigroup. Associated with the regular Dirichlet form $(\mathcal{E}, \mathcal{F})$ on $L^{2}(M ; \mu)$ is a $\mu$-symmetric Hunt process $X=\left\{X_{t}, t \geq 0 ; \mathbb{P}^{x}, x \in M \backslash \mathcal{N}\right\}$. Here $\mathcal{N} \subset M$ is a properly exceptional set for $(\mathcal{E}, \mathcal{F})$ in the sense that $\mathcal{N}$ is nearly Borel, $\mu(\mathcal{N})=0$ and $M_{\partial} \backslash \mathcal{N}$ is $X$-invariant. This Hunt process is unique up to a properly exceptional set - see [FOT, Theorem 4.2.8]. We fix $X$ and $\mathcal{N}$, and write $M_{0}=M \backslash \mathcal{N}$. The heat kernel associated with the semigroup $\left\{P_{t}\right\}_{t \geq 0}$ (if it exists) is a measurable function $p(t, x, y): M_{0} \times M_{0} \rightarrow(0, \infty)$ for every $t>0$, such that

$$
\begin{aligned}
\mathbb{E}^{x} f\left(X_{t}\right) & =P_{t} f(x)=\int p(t, x, y) f(y) \mu(d y) \quad \text { for all } x \in M_{0}, f \in L^{\infty}(M ; \mu), \\
p(t, x, y) & =p(t, y, x) \quad \text { for all } t>0, x, y \in M_{0}, \\
p(s+t, x, z) & =\int p(s, x, y) p(t, y, z) \mu(d y) \quad \text { for all } s>0, t>0, x, z \in M_{0} .
\end{aligned}
$$

While (1.5) only determines $p(t, x, \cdot) \mu$-a.e., using the Chapman-Kolmogorov equation (1.7) one can regularize $p(t, x, y)$ so that (1.5)-(1.7) hold for every point in $M_{0}$. See [BBCK, Theorem 3.1] and [GT, Section 2.2] for details. We call $p(t, x, y)$ the heat kernel on the metric measure Dirichlet space (or $M M D$ space) $(M, d, \mu, \mathcal{E})$. By (1.5), sometime we also call $p(t, x, y)$ the transition density function with respect to the measure $\mu$ for the process $X$.

Definition 1.2. Denote the ball centered at $x$ with radius $r$ by $B(x, r)$ and $\mu(B(x, r))$ by $V(x, r)$.

(i) We say that $(M, d, \mu)$ satisfies the volume doubling property (VD), if there exist constants $d_{2}, C_{\mu}>0$ such that for all $x \in M$ and $r>0$,

$$
\frac{V(x, R)}{V(x, r)} \leq C_{\mu}\left(\frac{R}{r}\right)^{d_{2}} \quad \text { for all } x \in M \text { and } 0<r \leq R .
$$


(ii) We say that $(M, d, \mu)$ satisfies the reverse volume doubling property (RVD), if there exist constants $d_{1}, c_{\mu}>0$ such that for all $x \in M$ and $0<r \leq R$,

$$
\frac{V(x, R)}{V(x, r)} \geq c_{\mu}\left(\frac{R}{r}\right)^{d_{1}}
$$

Note that VD condition 1.8 is equivalent to the existence of a positive constant $c_{1}$ so that

$$
V(x, 2 r) \leq c_{1} V(x, r) \quad \text { for all } x \in M \text { and } r>0,
$$

while RVD condition 1.9 is equivalent to the existence of positive constants $c_{2}>1$ and $l_{0}>0$ so that

$$
V\left(x, l_{0} r\right) \geq c_{2} V(x, r) \quad \text { for all } x \in M \text { and } r>0 .
$$

It is known that VD implies RVD if $M$ is connected and unbounded; e.g. see [GH, Proposition 5.1 and Corollary 5.3]. In this paper, we always assume that VD holds, and sometime we also assume RVD holds.

Set $\mathbb{R}_{+}:=[0, \infty)$. Let $\phi_{j}: \mathbb{R}_{+} \rightarrow \mathbb{R}_{+}$and $\phi_{c}:[1, \infty) \rightarrow \mathbb{R}_{+}$be strictly increasing continuous functions with $\phi_{j}(0)=\phi_{c}(0)=0, \phi_{j}(1)=\phi_{c}(1)=1$ and satisfying that there exist constants $c_{1, \phi_{j}}, c_{2, \phi_{j}}, c_{1, \phi_{c}}, c_{2, \phi_{c}}>0, \beta_{2, \phi_{j}} \geq \beta_{1, \phi_{j}}>0$ and $\beta_{2, \phi_{c}} \geq \beta_{1, \phi_{c}}>1$ such that

$$
\begin{aligned}
& c_{1, \phi_{j}}\left(\frac{R}{r}\right)^{\beta_{1, \phi_{j}}} \leq \frac{\phi_{j}(R)}{\phi_{j}(r)} \leq c_{2, \phi_{j}}\left(\frac{R}{r}\right)^{\beta_{2, \phi_{j}}} \quad \text { for all } 0<r \leq R, \\
& c_{1, \phi_{c}}\left(\frac{R}{r}\right)^{\beta_{1, \phi_{c}}} \leq \frac{\phi_{c}(R)}{\phi_{c}(r)} \leq c_{2, \phi_{c}}\left(\frac{R}{r}\right)^{\beta_{2, \phi_{c}}} \quad \text { for all } 0<r \leq R .
\end{aligned}
$$

Since $\beta_{1, \phi_{c}}>1$, we know from [BGT, Definition, p. 65; Definition, p. 66; Theorem 2.2 .4 and its remark, p. 73] that there exists a strictly increasing function $\bar{\phi}_{c}: \mathbb{R}_{+} \rightarrow \mathbb{R}_{+}$such that there is a constant $c_{1} \geq 1$ so that

$$
c_{1}^{-1} \phi_{c}(r) / r \leq \bar{\phi}_{c}(r) \leq c_{1} \phi_{c}(r) / r \quad \text { for all } r>0 .
$$

Clearly, by 1.10 and 1.11, there exist constants $c_{1, \bar{\phi}_{c}}, c_{2, \bar{\phi}_{c}}>0$ such that

$$
c_{1, \bar{\phi}_{c}}\left(\frac{R}{r}\right)^{\beta_{1, \phi_{c}}-1} \leq \frac{\bar{\phi}_{c}(R)}{\bar{\phi}_{c}(r)} \leq c_{2, \bar{\phi}_{c}}\left(\frac{R}{r}\right)^{\beta_{2, \phi_{c}}-1} \quad \text { for all } 0<r \leq R .
$$

Define

$\beta_{*}:=\sup \left\{\beta>0:\right.$ there is a constant $c_{*}>0$ so that $\phi_{j}(R) / \phi_{j}(r) \geq c_{*}(R / r)^{\beta}$ for $\left.0<r<R \leq 1\right\}$, and

$\beta^{*}:=\sup \left\{\beta>0:\right.$ there is a contant $c^{*}>0$ so that $\phi_{j}(R) / \phi_{j}(r) \geq c^{*}(R / r)^{\beta}$ for all $\left.R>r \geq 1\right\}$.

Throughout this paper, we assume that there is a constant $c_{0} \geq 1$ so that

$$
\phi_{c}(r) \leq c_{0} \phi_{j}(r) \text { on }[0,1] \text { if } \beta_{*}>1 \text { and } \phi_{c}(r) \leq c_{0} \phi_{j}(r) \text { on }(1, \infty) \text { if } \beta^{*}>1 .
$$

We point out that, by 1.10 with $\beta_{1, \phi_{c}}>1, \phi_{c}$ is not comparable to $\phi_{j}$ on $[0,1]$ when $\beta_{*} \leq 1$, and $\phi_{c}$ is not comparable to $\phi_{j}$ on $[1, \infty)$ when $\beta^{*} \leq 1$. 
In this paper, the function $\phi_{j}$ plays the role of scaling function in the jumping kernel; while $\phi_{c}$ is a scale function that should be intrinsically determined by $\phi_{j}$ and the metric measure space $(M, d, \mu)$, which will possibly appear in the expression of heat kernel estimates. However, we do not have a universal formula for $\phi_{c}$. For example, when the state space $M$ (such as $\mathbb{R}^{d}$ or a nice fractal) has a nice diffusion process, $\phi_{c}$ can be the scaling function of the diffusion in some cases but can also be a different scale function in some other cases; see Examples 1.1 and 5.2. In some cases, $\phi_{c}$ can just be $\phi_{j}$ on a part or the whole of $[0, \infty)$. To cover a wide spectrum of scenarios, in the formulation and characterization we allow $\phi_{c}$ to be any function that satisfies conditions 1.10 and 1.13 .

Given $\phi_{c}$ and $\phi_{j}$ as above, we set

$$
\phi(r):= \begin{cases}\phi_{j}(r) \mathbf{1}_{\left\{\beta_{*} \leq 1\right\}}+\phi_{c}(r) \mathbf{1}_{\left\{\beta_{*}>1\right\}} & \text { for } 0<r \leq 1 \\ \phi_{j}(r) \mathbf{1}_{\left\{\beta^{*} \leq 1\right\}}+\phi_{c}(r) \mathbf{1}_{\left\{\beta^{*}>1\right\}} & \text { for } r>1 .\end{cases}
$$

In view of the assumptions above, $\phi$ is strictly increasing on $\mathbb{R}_{+}$such that $\phi(0)=0, \phi(1)=1$ and there exist constants $c_{1, \phi}, c_{2, \phi}>0$ so that

$$
c_{1, \phi}\left(\frac{R}{r}\right)^{\beta_{1, \phi}} \leq \frac{\phi(R)}{\phi(r)} \leq c_{2, \phi}\left(\frac{R}{r}\right)^{\beta_{2, \phi}} \text { for all } 0<r \leq R,
$$

where $\beta_{1, \phi}=\beta_{1, \phi_{c}} \wedge \beta_{1, \phi_{j}}$ and $\beta_{2, \phi}=\beta_{2, \phi_{c}} \vee \beta_{2, \phi_{j}}$. Clearly, we have by 1.13 that

$$
\phi(r) \leq c_{0} \phi_{j}(r) \quad \text { for every } r \geq 0 .
$$

Denote by $\phi_{j}^{-1}(t), \phi_{c}^{-1}(t)$ and $\bar{\phi}_{c}^{-1}(t)$ the inverse functions of the strictly increasing functions $t \mapsto \phi_{j}(t), t \mapsto \phi_{c}(t)$ and $t \mapsto \bar{\phi}_{c}(t)$, respectively. Then the inverse function $\phi^{-1}$ of $\phi$ is given by

$$
\phi^{-1}(r):= \begin{cases}\phi_{j}^{-1}(r) \mathbf{1}_{\left\{\beta_{*} \leq 1\right\}}+\phi_{c}^{-1}(r) \mathbf{1}_{\left\{\beta_{*}>1\right\}} & \text { for } 0<r \leq 1, \\ \phi_{j}^{-1}(r) \mathbf{1}_{\left\{\beta^{*} \leq 1\right\}}+\phi_{c}^{-1}(r) \mathbf{1}_{\left\{\beta^{*}>1\right\}} & \text { for } r>1 .\end{cases}
$$

Throughout this paper, we will fix the notations for these functions $\phi_{c}, \phi_{j}, \phi$ and $\bar{\phi}_{c}$.

Definition 1.3. Let $\psi: \mathbb{R}_{+} \rightarrow \mathbb{R}_{+}$. We say $\mathrm{J}_{\psi}$ holds, if there exists a non-negative symmetric function $J(x, y)$ so that for all $x, y \in M$,

$$
J(d x, d y)=J(x, y) \mu(d x) \mu(d y)
$$

and

$$
\frac{c_{1}}{V(x, d(x, y)) \psi(d(x, y))} \leq J(x, y) \leq \frac{c_{2}}{V(x, d(x, y)) \psi(d(x, y))}
$$

We say that $\mathrm{J}_{\psi, \leq}\left(\right.$ resp. $\left.\mathrm{J}_{\psi, \geq}\right)$ if 1.16 holds and the upper bound (resp. lower bound) in (1.17) holds.

Note that, since $\phi(r) \leq c_{0} \phi_{j}(r)$ for all $r>0, \mathrm{~J}_{\phi_{j}, \leq}$ implies $\mathrm{J}_{\phi, \leq}$; that is, $\mathrm{J}_{\phi, \leq}$ is weaker than $\mathrm{J}_{\phi_{j}, \leq \cdot}$ 


\subsection{Main results}

To state our results about upper bounds and two-sided estimates on heat kernel precisely, we need a number of definitions. Some of them are taken from [CKW1].

For $f, g \in \mathcal{F}$, we define the carré du-Champ operator $\Gamma(f, g)$ for the pure jump Dirichlet form $(\mathcal{E}, \mathcal{F})$ by

$$
\Gamma(f, g)(d x)=\int_{y \in M}(f(x)-f(y))(g(x)-g(y)) J(d x, d y) .
$$

Clearly $\mathcal{E}(f, g)=\Gamma(f, g)(M)$.

Let $U \subset V$ be open sets of $M$ with $U \subset \bar{U} \subset V$, and let $\kappa \geq 1$. We say a non-negative bounded measurable function $\varphi$ is a $\kappa$-cut-off function for $U \subset V$, if $\varphi \geq 1$ on $U, \varphi=0$ on $V^{c}$ and $0 \leq \varphi \leq \kappa$ on $M$. Any 1-cut-off function is simply referred to as a cut-off function.

We now introduce the following cut-off Sobolev inequality $\operatorname{CSJ}(\phi)$ that controls the energy of cut-off functions.

Definition 1.4. Let $\mathcal{F}_{b}=\mathcal{F} \cap L^{\infty}(M, \mu)$. We say that condition $\operatorname{CSJ}(\phi)$ holds, if there exist constants $C_{0} \in(0,1]$ and $C_{1}, C_{2}>0$ such that for every $0<r \leq R$, almost all $x_{0} \in M$ and any $f \in \mathcal{F}$, there exists a cut-off function $\varphi \in \mathcal{F}_{b}$ for $B\left(x_{0}, R\right) \subset B\left(x_{0}, R+r\right)$ such that

$$
\begin{aligned}
& \int_{B\left(x_{0}, R+\left(1+C_{0}\right) r\right)} f^{2} d \Gamma(\varphi, \varphi) \\
& \leq C_{1} \int_{U \times U^{*}}(f(x)-f(y))^{2} J(d x, d y)+\frac{C_{2}}{\phi(r)} \int_{B\left(x_{0}, R+\left(1+C_{0}\right) r\right)} f^{2} d \mu,
\end{aligned}
$$

where $U=B\left(x_{0}, R+r\right) \backslash B\left(x_{0}, R\right)$ and $U^{*}=B\left(x_{0}, R+\left(1+C_{0}\right) r\right) \backslash B\left(x_{0}, R-C_{0} r\right)$.

Remark 1.5. (i) $\operatorname{CSJ}(\phi)$ for pure jump Dirichlet forms is first introduced in [CKW1] as a counterpart of $\operatorname{CSA}(\phi)$ for strongly local Dirichlet forms (see [AB, BB, BBK1]). As pointed out in [CKW1, Remark 1.6(ii)], the main difference between $\operatorname{CSJ}(\phi)$ and $\operatorname{CSA}(\phi)$ is that the integrals in the left hand side and in the second term of the right hand side of the inequality (1.18) are over $B\left(x_{0}, R+\left(1+C_{0}\right) r\right)$ instead of over $B\left(x_{0}, R+r\right)$ in [AB]. Note that the integral over $B\left(x_{0}, R+r\right)^{c}$ is zero in the left hand side of 1.18 for the case of strongly local Dirichlet forms. As we see from the approach of [CKW1] in the study of stability of heat kernel estimates for symmetric mixed stable-like processes, it is important to enlarge the ball $B\left(x_{0}, R+r\right)$ and integrate over $B\left(x_{0}, R+\left(1+C_{0}\right) r\right)$ rather than over $B\left(x_{0}, R+r\right)$.

(ii) Denote by $\mathcal{F}_{\text {loc }}$ the space of functions locally in $\mathcal{F}$; that is, $f \in \mathcal{F}_{\text {loc }}$ if and only if for any relatively compact open set $U \subset M$ there exists $g \in \mathcal{F}$ such that $f=g \mu$-a.e. on $U$. Since each ball is relatively compact and (1.18) uses the property of $f$ on $B\left(x_{0}, R+\left(1+C_{0}\right) r\right)$ only, $\operatorname{CSJ}(\phi)$ also holds for any $f \in \mathcal{F}_{l o c}$.

(iii) Consider the non-local symmetric Dirichlet form $(\mathcal{E}, \mathcal{F})$ on any geodesically complete Riemannian manifold that satisfies $\mathrm{VD}$ and $\mathrm{PI}\left(r^{2}\right)$ conditions so that $\mathrm{J}_{\phi_{j}}, \leq$ holds, where $\phi_{j}(r):=r^{\alpha} \vee r^{\beta}$ and $0<\alpha<2<\beta$. Then, by a similar argument as that for Example 1.1 given in the Appendix of this paper, $\operatorname{CSJ}(\phi)$ holds with $\phi(r)=r^{\alpha} \vee r^{2}$.

Following [GHH, Definition 1.11], we can adopt the generalized capacity inequality to the present setting, whose definition is much simpler than $\operatorname{CSJ}(\phi)$. 
Definition 1.6. We say that the generalized capacity inequality $\operatorname{Gcap}(\phi)$ holds, if there exist constants $\kappa \geq 1$ and $C>0$ such that for every $0<r<R, x_{0} \in M$ and any $u \in \mathcal{F}_{b}^{\prime}:=\{u+a$ : $\left.u \in \mathcal{F}_{b}, a \in \mathbb{R}\right\}$, there is a $\kappa$-cut-off function $\varphi \in \mathcal{F}$ for $B\left(x_{0}, R\right) \subset B\left(x_{0}, R+r\right)$ so that

$$
\mathcal{E}\left(u^{2} \varphi, \varphi\right) \leq \frac{C}{\phi(r)} \int_{B\left(x_{0}, R+r\right)} u^{2} d \mu .
$$

We next introduce the Faber-Krahn inequality, see [GT, Section 3.3] for more details. For $\alpha>0$, we define

$$
\mathcal{E}_{\alpha}(f, g)=\mathcal{E}(f, g)+\alpha \int_{M} f(x) g(x) \mu(d x) \quad \text { for } f, g \in \mathcal{F} .
$$

For any open set $D \subset M, \mathcal{F}_{D}$ is defined to be the $\mathcal{E}_{1}$-closure in $\mathcal{F}$ of $\mathcal{F} \cap C_{c}(D)$. Define

$$
\lambda_{1}(D)=\inf \left\{\mathcal{E}(f, f): f \in \mathcal{F}_{D} \text { with }\|f\|_{2}=1\right\},
$$

the bottom of the Dirichlet spectrum of $-\mathcal{L}$ on $D$.

Definition 1.7. The MMD space $(M, d, \mu, \mathcal{E})$ satisfies the Faber-Krahn inequality $\operatorname{FK}(\phi)$, if there exist positive constants $C$ and $p$ such that for any ball $B(x, r)$ and any open set $D \subset B(x, r)$,

$$
\lambda_{1}(D) \geq \frac{C}{\phi(r)}(V(x, r) / \mu(D))^{p} .
$$

Definition 1.8. We say that the (weak) Poincaré inequality $\mathrm{PI}(\phi)$ holds if there exist constants $C>0$ and $\kappa \geq 1$ such that for any ball $B_{r}=B(x, r)$ with $x \in M$ and for any $f \in \mathcal{F}_{b}$,

$$
\int_{B_{r}}\left(f-\bar{f}_{B_{r}}\right)^{2} d \mu \leq C \phi(r) \int_{B_{\kappa r} \times B_{\kappa r}}(f(y)-f(x))^{2} J(d x, d y),
$$

where $\bar{f}_{B_{r}}=\frac{1}{\mu\left(B_{r}\right)} \int_{B_{r}} f d \mu$ is the average value of $f$ on $B_{r}$.

Recall that $X=\left\{X_{t}\right\}$ is the Hunt process associated with the regular Dirichlet form $(\mathcal{E}, \mathcal{F})$ on $L^{2}(M ; \mu)$ with properly exceptional set $\mathcal{N}$, and $M_{0}:=M \backslash \mathcal{N}$. For a set $A \subset M$, define the exit time $\tau_{A}=\inf \left\{t>0: X_{t} \notin A\right\}$. We need the following definition on the exit time from balls.

Definition 1.9. (i) We say that $\mathrm{E}_{\phi}$ holds if there is a constant $c_{1}>1$ such that for all $r>0$ and all $x \in M_{0}$,

$$
c_{1}^{-1} \phi(r) \leq \mathbb{E}^{x}\left[\tau_{B(x, r)}\right] \leq c_{1} \phi(r) .
$$

We say that $\mathrm{E}_{\phi, \leq}$ (resp. $\mathrm{E}_{\phi, \geq}$ ) holds if the upper bound (resp. lower bound) in the inequality above holds.

(ii) We say $\mathrm{EP}_{\phi, \leq}$ holds if there is a constant $c>0$ such that for all $r, t>0$ and all $x \in M_{0}$,

$$
\mathbb{P}^{x}\left(\tau_{B(x, r)} \leq t\right) \leq \frac{c t}{\phi(r)} .
$$

Recall that the function $\bar{\phi}_{c}(r)$ is a strictly increasing function satisfying (1.11). For any $t>0$ and $x, y \in M_{0}$, set

$$
p^{(j)}(t, x, y):=\frac{1}{V\left(x, \phi_{j}^{-1}(t)\right)} \wedge \frac{t}{V(x, d(x, y)) \phi_{j}(d(x, y))}
$$

and

$$
p^{(c)}(t, x, y):=\frac{1}{V\left(x, \phi_{c}^{-1}(t)\right)} \exp \left(-\frac{d(x, y)}{\bar{\phi}_{c}^{-1}(t / d(x, y))}\right) .
$$


Definition 1.10. (i) We say that $\operatorname{HK}\left(\phi_{j}, \phi_{c}\right)$ holds if there exists a heat kernel $p(t, x, y)$ of the semigroup $\left\{P_{t}\right\}$ associated with $(\mathcal{E}, \mathcal{F})$, which has the following estimates for all $x, y \in M_{0}$,

$$
p(t, x, y) \asymp \begin{cases}\left\{\begin{array}{ll}
p^{(j)}(t, x, y) & \text { if } \beta_{*} \leq 1 \\
\left.\frac{1}{V\left(x, \phi_{c}^{-1}(t)\right)} \wedge\left(p^{(c)}(t, x, y)+p^{(j)}(t, x, y)\right)\right) & \text { if } \beta_{*}>1
\end{array} \text { for } 0<t \leq 1\right. \\
\left\{\begin{array}{ll}
p^{(j)}(t, x, y) & \text { if } \beta^{*} \leq 1 \\
\left.\frac{1}{V\left(x, \phi_{c}^{-1}(t)\right)} \wedge\left(p^{(c)}(t, x, y)+p^{(j)}(t, x, y)\right)\right) & \text { if } \beta^{*}>1
\end{array} \text { for } t>1 .\right.\end{cases}
$$

(ii) We say HK_ $\left(\phi_{j}, \phi_{c}\right)$ holds if the upper bound in 1.24 holds but the lower bound is replaced by the following statement: there are constants $c_{1}, c_{2}>0$ so that

$$
p(t, x, y) \geq c_{1} \begin{cases}\frac{1}{V\left(x, \phi^{-1}(t)\right)}, & d(x, y) \leq c_{2} \phi^{-1}(t) \\ \frac{t}{V(x, d(x, y)) \phi_{j}(d(x, y))}, & d(x, y) \geq c_{2} \phi^{-1}(t) .\end{cases}
$$

(iii) We say $\operatorname{UHK}\left(\phi_{j}, \phi_{c}\right)$ holds if the upper bound in (1.24) holds.

(iv) We say $\operatorname{UHKD}(\phi)$ holds if there is a constant $c>0$ such that for all $t>0$ and all $x \in M_{0}$,

$$
p(t, x, x) \leq \frac{c}{V\left(x, \phi^{-1}(t)\right)} .
$$

(v) We say $\mathrm{NL}(\phi)$ holds if there are constants $c_{1}, c_{2}>0$ such that for all $t>0$ and all $x, y \in M_{0}$ with $d(x, y) \leq c_{1} \phi^{-1}(t)$,

$$
p(t, x, y) \geq \frac{c_{2}}{V\left(x, \phi^{-1}(t)\right)} .
$$

(vi) For any open subset $D \subset M$, denote by $\left(P_{t}^{D}\right)_{t \geq 0}$ the (Dirichlet) semigroups of $\left(\mathcal{E}, \mathcal{F}_{D}\right)$, and by $p^{D}(t, x, y)$ the corresponding (Dirichlet) heat kernel. We say that a near diagonal lower bounded estimate for Dirichlet heat kernel $\mathrm{NDL}(\phi)$ holds, if there exist $\varepsilon \in(0,1)$ and $c_{1}>0$ such that for any $x_{0} \in M, r>0$ and $0<t \leq \phi(\varepsilon r)$,

$$
p^{B\left(x_{0}, r\right)}(t, x, y) \geq \frac{c_{1}}{V\left(x_{0}, \phi^{-1}(t)\right)} \quad \text { for } x, y \in B\left(x_{0}, \varepsilon \phi^{-1}(t)\right) \cap M_{0} .
$$

It is obvious that $\mathrm{NDL}(\phi)$ is stronger than $\mathrm{NL}(\phi)$, and $\mathrm{HK}_{-}\left(\phi_{j}, \phi_{c}\right)$ implies $\mathrm{NL}(\phi)$. On the other hand, under VD and (1.10), we can verify that $\mathrm{NL}(\phi)$ together with $\operatorname{UHK}\left(\phi_{j}, \phi_{c}\right)$ implies $\operatorname{NDL}(\phi)$; see the proof of [CKW4, Lemma 5.7].

We postpone discussions on the formulations of $\mathrm{HK}_{-}\left(\phi_{j}, \phi_{c}\right)$ and $\operatorname{HK}\left(\phi_{j}, \phi_{c}\right)$ to Subsection 1.4. The following are the two main results of this paper on heat kernel estimates.

Theorem 1.11. Assume that the metric measure space $(M, d, \mu)$ satisfies VD and RVD, and the functions $\phi_{c}$ and $\phi_{j}$ satisfy (1.10) and (1.13). Let $\phi(r)$ be defined by (1.14). The following are equivalent.

(1) $\mathrm{HK}_{-}\left(\phi_{j}, \phi_{c}\right)$.

(2) $\operatorname{UHK}\left(\phi_{j}, \phi_{c}\right), \mathrm{NL}(\phi)$ and $\mathrm{J}_{\phi_{j}}$.

(3) $\operatorname{UHKD}(\phi), \operatorname{NDL}(\phi)$ and $\mathrm{J}_{\phi_{j}}$.

(4) $\mathrm{PI}(\phi), \mathrm{J}_{\phi_{j}}$ and $\mathrm{E}_{\phi}$. 
(5) $\operatorname{PI}(\phi), \mathrm{J}_{\phi_{j}}$ and $\operatorname{Gcap}(\phi)$.

(6) $\mathrm{PI}(\phi), \mathrm{J}_{\phi_{j}}$ and $\operatorname{CSJ}(\phi)$.

If, in additional, $(M, d, \mu)$ is connected and satisfies the chain condition, then each above assertion is equivalent to

(7) $\operatorname{HK}\left(\phi_{j}, \phi_{c}\right)$.

In the process of establishing Theorem 1.11, we also obtain the following characterizations for $\operatorname{UHK}\left(\phi_{j}, \phi_{c}\right)$. In the following, we say $(\mathcal{E}, \mathcal{F})$ is conservative if its associated Hunt process $X$ has infinite lifetime. This is equivalent to $P_{t} 1=1$ on $M_{0}$ for every $t>0$. It follows from CKW1, Proposition 3.1] that $\mathrm{NL}(\phi)$ implies that $(\mathcal{E}, \mathcal{F})$ is conservative. Thus any equivalent statement of Theorem 1.11 implies that the process $X$ is conservative.

Theorem 1.12. Assume that the metric measure space $(M, d, \mu)$ satisfies VD and RVD, and that the functions $\phi_{c}$ and $\phi_{j}$ satisfy (1.10) and (1.13). Let $\phi(r)$ be defined by (1.14). Then the following are equivalent:

(1) $\operatorname{UHK}\left(\phi_{j}, \phi_{c}\right)$ and $(\mathcal{E}, \mathcal{F})$ is conservative.

(2) $\operatorname{UHKD}(\phi), \mathrm{J}_{\phi_{j}, \leq}$ and $\mathrm{E}_{\phi}$.

(3) $\mathrm{FK}(\phi), \mathrm{J}_{\phi_{j}, \leq}$ and $\mathrm{E}_{\phi}$.

(4) $\mathrm{FK}(\phi), \mathrm{J}_{\phi_{j}, \leq}$ and $\operatorname{Gcap}(\phi)$.

(5) $\mathrm{FK}(\phi), \mathrm{J}_{\phi_{j}, \leq}$ and $\operatorname{CSJ}(\phi)$.

We emphasize that the above two theorems are equivalent characterizations and stability results. It is possible that none of the statements hold with a bad selection of $\phi_{c}$.

We point out that $\operatorname{UHK}\left(\phi_{j}, \phi_{c}\right)$ alone does not imply the conservativeness of the associated Dirichlet form $(\mathcal{E}, \mathcal{F})$. See [CKW1, Proposition 3.1 and Remark 3.2] for more details. Under VD, RVD and (1.10), NDL $(\phi)$ implies $\mathrm{E}_{\phi}$ (e.g., see [CKW2, Proposition 3.5(2)]), and so (3) in Theorem 1.11 is stronger than (2) in Theorem 1.12. We also note that RVD is only used in the implications that $\operatorname{UHKD}(\phi) \Longrightarrow \mathrm{FK}(\phi)$ and $\mathrm{PI}(\phi) \Longrightarrow \mathrm{FK}(\phi)$; see Proposition 2.1. In particular, $(5) \Longrightarrow(1)$ in Theorem 1.12 holds true under VD and $(1.10)$.

Let $Z:=\left\{V_{s}, X_{s}\right\}_{s \geq 0}$ be the space-time process corresponding to $X$, where $V_{s}=V_{0}-s$. The law of the space-time process $Z$ starting from $(t, x)$ will be denoted by $\mathbb{P}^{(t, x)}$. For every open subset $D$ of $[0, \infty) \times M$, define $\tau_{D}=\inf \left\{s>0: Z_{s} \notin D\right\}$.

Definition 1.13. (i) We say that a Borel measurable function $u(t, x)$ on $[0, \infty) \times M$ is parabolic (or caloric) on $D=(a, b) \times B\left(x_{0}, r\right)$ for the process $X$ if there is a properly exceptional set $\mathcal{N}_{u}$ associated with the process $X$ so that for every relatively compact open subset $U$ of $D, u(t, x)=\mathbb{E}^{(t, x)} u\left(Z_{\tau_{U}}\right)$ for every $(t, x) \in U \cap\left([0, \infty) \times\left(M \backslash \mathcal{N}_{u}\right)\right)$.

(ii) We say that the parabolic Harnack inequality $(\mathrm{PHI}(\phi))$ holds for the process $X$, if there exist constants $0<C_{1}<C_{2}<C_{3}<C_{4}, C_{5}>1$ and $C_{6}>0$ such that for every $x_{0} \in M$, $t_{0} \geq 0, R>0$ and for every non-negative function $u=u(t, x)$ on $[0, \infty) \times M$ that is parabolic on cylinder $Q\left(t_{0}, x_{0}, \phi\left(C_{4} R\right), C_{5} R\right):=\left(t_{0}, t_{0}+\phi\left(C_{4} R\right)\right) \times B\left(x_{0}, C_{5} R\right)$,

$$
\operatorname{ess} \sup _{Q_{-}} u \leq C_{6} \operatorname{ess} \inf _{Q_{+}} u,
$$

where $Q_{-}:=\left(t_{0}+\phi\left(C_{1} R\right), t_{0}+\phi\left(C_{2} R\right)\right) \times B\left(x_{0}, R\right)$ and $Q_{+}:=\left(t_{0}+\phi\left(C_{3} R\right), t_{0}+\phi\left(C_{4} R\right)\right) \times$ $B\left(x_{0}, R\right)$. 
The following definition was introduced in BBK2 in the setting of graphs, and then extended in CKK2] to the general setting of metric measure spaces.

Definition 1.14. We say that UJS holds if there is a symmetric function $J(x, y)$ so that 1.16 ) holds, and there is a constant $c>0$ such that for any $x, y \in M$ and $0<r \leq d(x, y) / 2$,

$$
J(x, y) \leq \frac{c}{V(x, r)} \int_{B(x, r)} J(z, y) \mu(d z) .
$$

The result below gives us the stable characterization of parabolic Harnack inequalities, as well as the relation between parabolic Harnack inequalities and two-sided heat kernel estimates.

Theorem 1.15. Suppose that the metric measure space $(M, d, \mu)$ satisfies VD and RVD, and that the functions $\phi_{c}$ and $\phi_{j}$ satisfy (1.10) and (1.13). Let $\phi(r)$ be defined by (1.14). Then

$$
\mathrm{PHI}(\phi) \Longleftrightarrow \mathrm{PI}(\phi)+\mathrm{J}_{\phi, \leq}+\mathrm{CSJ}(\phi)+\mathrm{UJS} \text {. }
$$

Consequently,

$$
\mathrm{HK}_{-}\left(\phi_{j}, \phi_{c}\right) \Longleftrightarrow \mathrm{PHI}(\phi)+\mathrm{J}_{\phi_{j}} .
$$

If, additionally, the metric measure space $(M, d, \mu)$ is connected and satisfies the chain condition, then

$$
\operatorname{HK}\left(\phi_{j}, \phi_{c}\right) \Longleftrightarrow \operatorname{PHI}(\phi)+\mathrm{J}_{\phi_{j}} .
$$

Just like in [CKW2, CKW4], we could get more equivalent statements for $\mathrm{PHI}(\phi)$ in Theorem 1.15. We are not doing it here for the sake of space consideration.

We emphasize again that the connectedness and the chain condition of the underlying metric measure space $(M, d, \mu)$ is only used to derive optimal lower bounds of off-diagonal estimates for the heat kernel (i.e., from $\operatorname{HK}_{-}\left(\phi_{j}, \phi_{c}\right)$ to $\left.\operatorname{HK}\left(\phi_{j}, \phi_{c}\right)\right)$. For other statements in the three main results above, the metric measure space $(M, d, \mu)$ is only assumed to satisfy the general VD and RVD; that is, neither do we assume $M$ to be connected nor $(M, d)$ to be geodesic. Furthermore, we do not assume the uniform comparability of volume of balls; that is, we do not assume the existence of a non-decreasing function $V$ on $[0, \infty)$ with $V(0)=0$ so that $\mu(B(x, r)) \asymp V(r)$ for all $x \in M$ and $r>0$.

\subsection{Remarks and discussions}

In this subsection, we comment on the formulations of $\operatorname{HK}_{-}\left(\phi_{j}, \phi_{c}\right)$ and $\operatorname{HK}\left(\phi_{j}, \phi_{c}\right)$, and discuss the relations of the main results to those in the literature. Recall that the function $\phi$ is defined by $(1.14)$.

Remark 1.16. (i) The scale function $\phi_{c}$ plays a role in the definitions of $\operatorname{HK}\left(\phi_{j}, \phi_{c}\right), \mathrm{HK}_{-}\left(\phi_{j}\right.$, $\left.\phi_{c}\right), \operatorname{UHK}\left(\phi_{j}, \phi_{c}\right), \operatorname{UHKD}(\phi), \operatorname{NL}(\phi)$ and $\operatorname{NDL}(\phi)$ only for $t \leq 1$ when $\beta_{*}>1$ and for $t \geq 1$ when $\beta^{*}>1$. The cut-off time 1 here is not important. It can be replaced by any fixed constant $T>0$. Furthermore, it follows from Theorem 1.15 that HK_ $\left(\phi_{j}, \phi_{c}\right)$ (and so $\operatorname{HK}\left(\phi_{j}, \phi_{c}\right)$ ) is stronger than $\operatorname{PHI}(\phi)$, which in turn yields the Hölder regularity of parabolic functions; see the proof of [CKW4, Theorem 1.17]. In particular, this implies that if $\operatorname{HK}_{-}\left(\phi_{j}, \phi_{c}\right)$ (respectively, $\left.\operatorname{HK}\left(\phi_{j}, \phi_{c}\right)\right)$ holds, then it can be strengthened to hold for all $x, y \in M$, and consequently the Hunt process $X$ can be refined to start from every point in $M$. 
(ii) Note that when $d(x, y) \leq \phi_{c}^{-1}(t)$,

$$
p^{(c)}(t, x, y) \simeq \frac{1}{V\left(x, \phi_{c}^{-1}(t)\right)}
$$

and that when $d(x, y) \geq \phi_{c}^{-1}(t)$, for every $k \geq 1$,

$$
\begin{aligned}
p^{(c)}(t, x, y) & \leq \frac{c_{1}}{V(x, d(x, y))} \frac{V(x, d(x, y))}{V\left(x, \phi_{c}^{-1}(t)\right)} \exp \left(-c_{2}\left(\frac{\phi_{c}(d(x, y))}{t}\right)^{1 /\left(\beta_{2, \phi_{c}}-1\right)}\right) \\
& \leq \frac{c_{3}}{V(x, d(x, y))}\left(\frac{\phi_{c}(d(x, y))}{t}\right)^{d_{2} / \beta_{1, \phi_{c}}} \exp \left(-c_{2}\left(\frac{\phi_{c}(d(x, y))}{t}\right)^{1 /\left(\beta_{2, \phi_{c}}-1\right)}\right) \\
& \leq \frac{c_{4}(k)}{V(x, d(x, y))} \frac{t^{k}}{\phi_{c}(d(x, y))^{k}},
\end{aligned}
$$

where in the first inequality we used 1.11 and 1.12 , in the second inequality we used $\mathrm{VD}$ and 1.10, and the last inequality is a consequence of the elementary inequality

$$
r^{k+d_{2} / \beta_{1, \phi_{c}}} \exp \left(-c_{2} r^{1 /\left(\beta_{2, \phi_{c}}-1\right)}\right) \leq c_{5}(k), \quad r \geq 1 .
$$

So in particular, we have by 1.27 and 1.28 with $k=1$,

$$
p^{(c)}(t, x, y) \preceq \frac{1}{V\left(x, \phi_{c}^{-1}(t)\right)} \wedge \frac{t}{V(x, d(x, y)) \phi_{c}(d(x, y))} \quad \text { on }(0, \infty) \times M_{0} \times M_{0} .
$$

Hence when $\beta^{*}>1$ and $\phi_{c}=\phi_{j}$ on $[1, \infty)$, we have

$$
p^{(c)}(t, x, y) \preceq \frac{1}{V\left(x, \phi_{j}^{-1}(t)\right)} \wedge \frac{t}{V(x, d(x, y)) \phi_{j}(d(x, y))} \quad \text { on }[1, \infty) \times M_{0} \times M_{0} .
$$

Consequently, in this case,

$$
\frac{1}{V\left(x, \phi_{c}^{-1}(t)\right)} \wedge\left(p^{(c)}(t, x, y)+p^{(j)}(t, x, y)\right) \asymp p^{(j)}(t, x, y) \quad \text { on }[1, \infty) \times M_{0} \times M_{0} .
$$

On the other hand, note that by $(1.10)$, there is some $k \geq 1$ so that $\phi_{c}(r)^{k} \geq c_{6} \phi_{j}(r)$ on $[1, \infty)$. Thus it follows from 1.28 that for $t \in(0,1]$ and $d(x, y) \geq 1$,

$$
p^{(c)}(t, x, y) \leq \frac{c_{7} t}{V(x, d(x, y)) \phi_{j}(d(x, y))} .
$$

This together with (1.27) and 1.29 for $d(x, y) \leq 1$ shows that in the case of $\beta_{*}>1$ and $\phi_{c}=\phi_{j}$ on $[0,1]$,

$$
\frac{1}{V\left(x, \phi_{c}^{-1}(t)\right)} \wedge\left(p^{(c)}(t, x, y)+p^{(j)}(t, x, y)\right) \asymp p^{(j)}(t, x, y) \quad \text { on }(0,1] \times M_{0} \times M_{0} .
$$

Hence when $\phi=\phi_{j}$ on $[0, \infty)$ (that is, when $\phi_{c}=\phi_{j}$ on $[0,1]$ if $\beta_{*}>1$ and $\phi_{c}=\phi_{j}$ on $(1, \infty)$ if $\left.\beta^{*}>1\right), \operatorname{HK}\left(\phi_{j}, \phi_{c}\right)$ is just the heat kernel estimate $p^{(j)}(t, x, y)$. Thus in the case of $\phi=\phi_{j}$ on $[0, \infty)$, Theorems 1.11 and 1.12 are essentially the main results (Theorems 1.13 and 1.15) of [CKW1, and Theorem 1.15 is an essential part of [CKW2, Theorem 1.20]. We note that by the proof of [CKW1, Lemma 4.1], $\mathrm{J}_{\phi_{j}, \geq}$ implies $\operatorname{PI}\left(\phi_{j}\right)$ so in the case of $\phi=\phi_{j}$ on $[0, \infty)$, we can drop condition $\operatorname{PI}(\phi)$ from the statement of Theorem 1.11 . 
(iii) When $\phi(r)=\phi_{c}(r)$ on $[0, \infty)$ (that is, when $\beta_{*} \wedge \beta^{*}>1$, and $\phi_{c}(r)$ is a strictly increasing function satisfying (1.10) and 1.13$), \mathrm{HK}_{-}\left(\phi_{j}, \phi_{c}\right)$ and $\operatorname{HK}\left(\phi_{j}, \phi_{c}\right)$ are just $\operatorname{HK}(\Phi, \psi)$ and $\operatorname{SHK}(\Phi, \psi)$ in [BKKL2, Definition 2.8] with

$$
\psi=\phi_{j} \quad \text { and } \quad \Phi=\phi_{c} .
$$

In this case, our Theorems 1.11 and 1.12 have also been independently obtained in BKKL2, Theorem 2.14, Corollary 2.15, Theorem 2.17 and Corollary 2.18]. See Remark 1.18 below for more information.

(iv) When $\phi(r)=\phi_{c}(r) \mathbf{1}_{[0,1]}+\phi_{j}(r) \mathbf{1}_{(1, \infty)}$ (that is, when $\beta_{*}>1$ and either $\beta^{*} \leq 1$ or $\beta^{*}>1$ with $\phi_{c}(r)=\phi_{j}(r)$ for all $\left.r \in[1, \infty)\right)$, by (1.27) and $(1.29), \operatorname{HK}\left(\phi_{j}, \phi_{c}\right)$ is reduced into

$$
p(t, x, y) \asymp \begin{cases}\left.\frac{1}{V\left(x, \phi_{c}^{-1}(t)\right)} \wedge\left(p^{(c)}(t, x, y)+p^{(j)}(t, x, y)\right)\right), & 0<t \leq 1, \\ p^{(j)}(t, x, y), & t>1 .\end{cases}
$$

In this case $\operatorname{HK}\left(\phi_{j}, \phi_{c}\right)$ is of the same form as that of $\operatorname{HK}\left(\phi_{c}, \phi_{j}\right)$ in CKW4 for symmetric diffusions with jumps, or equivalent, for symmetric Dirichlet forms that contain both the strongly local part and the pure jump part; see [CKW4, Definition 1.11 and Remark 1.12] for details. However, they are quite different. In [CKW4 the function $\phi_{c}$ in $\operatorname{HK}\left(\phi_{c}, \phi_{j}\right)$ is the scaling function of the diffusion (i.e. the strongly local part of Dirichlet forms), while in the present paper the function $\phi_{c}$ in $\operatorname{HK}\left(\phi_{j}, \phi_{c}\right)$ is determined by $\phi_{j}$ and the underlying metric measure space $(M, d, \mu)$.

(v) We note that the expression of $\operatorname{HK}\left(\phi_{j}, \phi_{c}\right)$ takes different form depending on whether $\beta_{*} \leq 1$ (respectively, $\beta^{*} \leq 1$ ) or not. This is because when $\beta_{*}>1$ (respectively, $\beta^{*}>1$ ), the heat kernel estimate may involve another function $\phi_{c}$ that is intrinsically determined by $\phi_{j}$ but we do not have a generic formula for it under our general setting. However it does not necessarily mean that the heat kernel estimates for $p(t, x, y)$ has a phase transition exactly at $\beta_{*}=1$ or $\beta^{*}=1$. For example, suppose $(M, d, \mu)$ is a connected metric measure space satisfying the chain condition on which there is a symmetric diffusion process enjoying the heat kernel estimate (1.1) with $\beta>1$ as in Example 1.1. Consider a pure jump symmetric process $Y$ on this space whose jumping density has bounds $(1.2)$ with $\alpha_{1}<\beta$ and $\alpha_{2}>0$. Clearly, $\beta_{*}=\alpha_{1}$ and $\beta^{*}=\alpha_{2}$. When $\alpha_{2}<\beta$, the transition density function $p(t, x, y)$ of $Y$ has estimates 1.22 with $\phi_{j}(r)=r^{\alpha_{1}} \mathbf{1}_{\{0<r \leq 1\}}+r^{\alpha_{2}} \mathbf{1}_{\{r>1\}}$ as in CKW1, whereas for $\alpha_{2}>\beta, p(t, x, y)$ satisfies (1.4) as in Example 1.1. Hence for this example, phase transition for the expression of heat kernel estimates for $p(t, x, y)$ occurs at $\alpha_{2}=\beta$.

In (ii)-(iv) of Remark 1.16, we have discussed the form of $\operatorname{HK}\left(\phi_{j}, \phi_{c}\right)$ for the cases of $\phi(r)=$ $\phi_{j}(r)$ on $[0, \infty), \phi(r)=\phi_{c}(r)$ on $[0, \infty)$, and $\phi(r)=\phi_{c}(r) \mathbf{1}_{[0,1]}+\phi_{j}(r) \mathbf{1}_{(1, \infty)}$. We now discuss the remaining case of $\phi(r)$.

Remark 1.17. This remark is concerned with the case that $\phi(r)=\phi_{j}(r) \mathbf{1}_{[0,1]}+\phi_{c}(r) \mathbf{1}_{(1, \infty)}$. It consists of two subcases of $\beta^{*}>1$ where either $\beta_{*} \leq 1$ or $\beta_{*}>1$ with $\phi_{c}(r)=\phi_{j}(r)$ for all $r \in(0,1]$.

(i) In this case, we can rewrite the expression of $\operatorname{HK}\left(\phi_{j}, \phi_{c}\right)$ in the following way. For $0<t \leq 1$,

$$
p(t, x, y) \simeq p^{(j)}(t, x, y) \simeq \begin{cases}\frac{1}{V\left(x, \phi_{j}^{-1}(t)\right)}, & d(x, y) \leq c_{1} \phi_{j}^{-1}(t), \\ \frac{t}{V(x, d(x, y)) \phi_{j}(d(x, y))}, & d(x, y) \geq c_{1} \phi_{j}^{-1}(t) .\end{cases}
$$




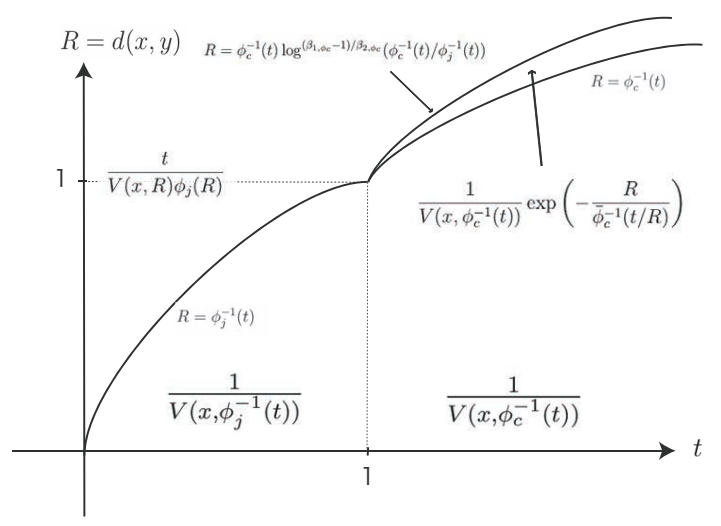

Figure 1: Dominant term in the heat kernel estimates $\operatorname{HK}\left(\phi_{j}, \phi_{c}\right)$ for $p(t, x, y)$ for the case in Remark 1.17 .

For $t \geq 1$,

$$
\begin{aligned}
p(t, x, y) & \asymp \frac{1}{V\left(x, \phi_{c}^{-1}(t)\right)} \wedge\left(p^{(c)}(t, x, y)+p^{(j)}(t, x, y)\right) \\
& \asymp \begin{cases}\frac{1}{V\left(x, \phi_{c}^{-1}(t)\right)}, & d(x, y) \leq c_{2} \phi_{c}^{-1}(t), \\
\frac{t}{V(x, d(x, y)) \phi_{j}(d(x, y))}+\frac{1}{V\left(x, \phi_{c}^{-1}(t)\right)} \exp \left(-\frac{d(x, y)}{\bar{\phi}_{c}^{-1}(t / d(x, y))}\right), & d(x, y) \geq c_{2} \phi_{c}^{-1}(t) .\end{cases}
\end{aligned}
$$

See the argument in Remark 1.16(ii). In particular, for $t \in(0,1]$, the heat kernel estimates $\operatorname{HK}\left(\phi_{c}, \phi_{j}\right)$ are completely dominated by the jumping kernel for the associated Dirichlet form $(\mathcal{E}, \mathcal{F})$. For $t \geq 1$, we have the following more explicit expression of $\operatorname{HK}\left(\phi_{j}, \phi_{c}\right)$ :

$$
p(t, x, y) \asymp \begin{cases}\frac{1}{V\left(x, \phi_{c}^{-1}(t)\right)}, & d(x, y) \leq c_{2} \phi_{c}^{-1}(t), \\ \frac{1}{V\left(x, \phi_{c}^{-1}(t)\right)} \exp \left(-\frac{d(x, y)}{\bar{\phi}_{c}^{-1}(t / d(x, y))}\right), & c_{2} \phi_{c}^{-1}(t) \leq d(x, y) \leq t_{*}, \\ \frac{t}{V(x, d(x, y)) \phi_{j}(d(x, y))}, & d(x, y) \geq t_{*},\end{cases}
$$

where $t_{*}$ satisfies that

$$
c_{3} \phi_{c}^{-1}(t) \log \left(\beta_{1, \phi_{c}}-1\right) / \beta_{2, \phi_{c}}\left(\phi_{c}^{-1}(t) / \phi_{j}^{-1}(t)\right) \leq t_{*} \leq c_{4} \phi_{c}^{-1}(t) \log { }^{\left(\beta_{2, \phi_{c}}-1\right) / \beta_{1, \phi_{c}}}\left(\phi_{c}^{-1}(t) / \phi_{j}^{-1}(t)\right),
$$

and $\beta_{1, \phi_{c}}$ and $\beta_{2, \phi_{c}}$ are given in 1.10 . See the proof of Proposition 3.5 for more details. In this case, one can check that only the information of $\phi_{c}$ on $[1, \infty)$ is actually needed for the expression of $\operatorname{HK}\left(\phi_{j}, \phi_{c}\right)$ because $t / d(x, y) \geq c_{3}>0$ holds when $t \geq 1$ and $c_{2} \phi_{c}^{-1}(t) \leq$ $d(x, y) \leq t_{*}$. Figure 1 indicates in this case which term is the dominant one for the estimate of $p(t, x, y)$ in each region.

(ii) The definition of $\operatorname{HK}\left(\phi_{j}, \phi_{c}\right)$ in this case is different from $\operatorname{HK}\left(\phi_{c}, \phi_{j}\right)$ in [CKW4 for symmetric diffusions with jumps. Denote by the heat kernel by $\bar{p}(t, x, y)$ in the case of symmetric diffusion with jumps. We say $\operatorname{HK}\left(\phi_{c}, \phi_{j}\right)$ holds if 1.30 holds. Thus the expressions for $\operatorname{HK}\left(\phi_{j}, \phi_{c}\right)$ for symmetric jump processes with lighter jumping tails and $\operatorname{HK}\left(\phi_{c}, \phi_{j}\right)$ for symmetric diffusions with jumps are exactly switched over the time interval $(0,1]$ and $(1, \infty)$. The reason for this difference is as follows. For diffusions with jumps, due to the heavy tail property of jump phenomenon, the behaviors of the processes for large time are 
dominated by the pure jump part, and the behaviors for small time enjoy the continuous nature from diffusions as well as some interactions with jumps. For symmetric pure jumps processes with lighter tails considered in this case, the small time behavior of the processes are controlled by the jumping kernels; however, since large jumps of the associated process is so light (as a typical example, one can consider symmetric jump processes on $\mathbb{R}^{d}$ whose associated jumping measure has finite second moments), it essentially also yields the diffusive property of the process when time becomes large.

(iii) The lower bound in $\mathrm{HK}_{-}\left(\phi_{j}, \phi_{c}\right)$ can be expressed more explicitly; namely, there are constants $c_{1}, c_{2}>0$ so that

$$
p(t, x, y) \geq c_{1} \begin{cases}p^{(j)}(t, x, y), & 0<t \leq 1 \\ \frac{1}{V\left(x, \phi_{c}^{-1}(t)\right)}, & t \geq 1, d(x, y) \leq c_{2} \phi_{c}^{-1}(t), \\ \frac{t}{V(x, d(x, y)) \phi_{j}(d(x, y))}, & t \geq 1, d(x, y) \geq c_{2} \phi_{c}^{-1}(t) .\end{cases}
$$

Remark 1.18. We make some remarks on the relations of our main results to those in [BKKL2].

(i) At the time we were finalizing this paper, we noticed an arXiv preprint BKKL2 on a similar topic as (part of) this paper; see Remark 1.16(iii). The paper [BKKL2] is a partial extension of [CKW1] as well as [BKKL1]. Paper [BKKL1] studies heat kernel estimates for symmetric jump processes on $\mathbb{R}^{d}$ with jumping kernels of mixed polynomial growth rate not necessarily less than 2 . It is strongly motivated by [Mi], which studied heat kernel estimates for subordinate Brownian motions with scaling order not necessarily strictly less than 2, and uses some techniques from [CKW1]. The heat kernel estimate in [BKKL1] takes the form of

$$
\frac{1}{V\left(x, \phi_{c}^{-1}(t)\right)} \wedge\left(p^{(c)}(t, x, y)+p^{(j)}(t, x, y)\right)
$$

with $V(x, r)=r^{d}$ and $\phi_{c}(r):=r^{2} / \int_{0}^{r} s / \phi_{j}(s) d s$. Paper [BKKL2] extends above formation of heat kernel estimates to metric measure spaces with more general scaling function $\phi_{c}$ and studies its stable characterizations in the sprit of [CKW1. However the formulation of (1.31) excludes some interesting cases such as those considered in Example 1.1, as it essentially requires the lower scaling index of $\phi_{j}$ on $[0,1]$ to be strictly larger than 1 . It does not cover some cases studied in CKW1 either when the global lower scaling index of $\phi_{j}$ is no larger than 1. See part (ii) below for more information.

The advantage of our setting and our approach is that we start with two scaling functions $\phi_{j}$ and $\phi_{c}$. This starting point is motivated by Example 1.1, and is quite natural. This allows us to treat the cases of $0<r \leq 1$ and $r>1$ separably with possibly different scaling indices. It also allows us to incorporate the heat kernel formulation (1.31) considered in [BKKL1, BKKL2]. The significance of this viewpoint is further illustrated by Example 5.2 where the local lower scaling index of the scale function $\phi_{j}$ on $(1, \infty)$ is strictly larger than 1, while the local lower scaling index of the scale function $\phi_{j}$ on $(0,1)$ can take any value in $(0,2)$. Both Example 1.1 and Example 5.2 fall outside the main settings of [BKKL1, BKKL2]. However, see a less precise heat kernel estimates in [BKKL2, Theorem 2.19].

A key step in our approach is Proposition 3.5, whose proof is the most difficult part of our paper as mentioned above. The proof of Proposition 3.5 is strongly motivated by that of 
CKW4, Proposition 4.6] for symmetric diffusion with jumps. (Indeed, the main results of this paper indicates the similar philosophy of heat kernel estimates between diffusions with jumps in CKW4 and pure jump Markov processes having lighter tails considered in this paper under the viewpoint that time interval $t \leq 1$ and $t>1$ switched.) The counterpart of our Proposition 3.5 in BKKL2] is Theorem 2.13 there. Actually, BKKL2, Theorem 2.13 ] plays a crucial role in all the main results in [BKKL2]. With this at hand, the authors fully used approaches from [CKW1, CKW2] to establish the stability characterizations of heat kernel estimates [BKKL2]; see Theorem 2.14 and Corollary 2.15; Theorem 2.17 and Corollary 2.18 in that paper for more details. The proof of [BKKL2, Theorem 2.13] is based on that of [BKKL1, Theorem 4.5], which makes full use of the property that the global lower weak scaling index of $\phi_{c}$ (or $\Phi$ in their notation) is strictly larger than 1 . Though this self-improvement approach looks a little similar, the proof of Proposition 3.5 of this paper is much more delicate as our setting is more general. Our approach works for the setting of [BKKL1] and beyond. Moreover, our heat kernel estimate $\operatorname{HK}\left(\phi_{j}, \phi_{c}\right)$ is more concise and explicit, see Remark 1.17(i). Furthermore, our approach also yields the stable characterizations of parabolic Harnack inequalities, which are useful as indicated in Example 5.3. Parabolic Harnack inequalities are not studied in BKKL2].

(ii) It is assumed in BKKL2 that $\Phi(r) \leq c_{1} \psi(r)$ for all $r \geq 0$ and that the global lower scaling index of $\Phi$ is strictly larger than 1. It follows that $\beta^{*}>1$ for $\phi_{j}:=\psi$. Although it is not mentioned explicitly in the corresponding results in BKKL2, for $\operatorname{HK}(\Phi, \psi)$ and $\operatorname{SHK}(\Phi, \psi)$ to hold, the lower scaling index $\beta_{*}$ has to be strictly larger than 1 as well, if $\inf _{x \in M} V(x, 1)>0$ and there are some constants $c_{1}>0$ and $\beta_{2} \in(0,2)$ so that

$$
\phi_{j}(R) / \phi_{j}(r) \leq c_{1}(R / r)^{\beta_{2}} \text { for } 0<r<R \leq 1 .
$$

Below is the reasoning. Using the notation of this paper (that is, $\psi=\phi_{j}$ and $\Phi=\phi_{c}$ ), define

$$
\bar{\phi}_{j}(r)=\phi_{j}(r) \mathbf{1}_{[0,1]}(r)+r^{\beta^{*} \wedge(3 / 2)} \mathbf{1}_{[1, \infty)}(r) .
$$

Clearly there is a constant $c_{2}>0$ so that $\bar{\phi}_{j}(r) \leq c_{2} \phi_{j}(r)$ on $[0, \infty)$ and

$$
J(x, y) \leq \frac{c_{2}}{V(x, d(x, y)) \bar{\phi}_{j}(d(x, y))} \quad \text { for } d(x, y) \geq 1 .
$$

Let

$$
\bar{J}(x, y):=J(x, y) \mathbf{1}_{\{d(x, y) \leq 1\}}+\frac{c_{2}}{V(x, d(x, y)) \bar{\phi}_{j}(d(x, y))} \mathbf{1}_{\{d(x, y)>1\}} .
$$

Clearly, $J(x, y) \leq \bar{J}(x, y)$ and $\|\bar{J}-J\|_{\infty} \leq c_{3} / \inf _{x \in M} V(x, 1)<\infty$. By the calculation in [CKW1, Lemma 2.1], one can show that

$\left.\mathcal{J}(x):=\int_{M}(\bar{J}(x, y)-J(x, y))\right) \mu(d y) \leq \int_{M} \frac{c_{2}}{V(x, d(x, y)) \bar{\phi}_{j}(d(x, y))} \mathbf{1}_{\{d(x, y)>1\}} \mu(d x) \leq c_{4}$

for every $x \in M$. Denote by $\bar{p}(t, x, y)$ the transition density function for the symmetric Hunt process $\bar{X}$ with jumping kernel $\bar{J}(x, y)$. It follows from [BGK, Lemma 3.1] and [BBCK, Lemma 3.6] that

$$
e^{-c_{4} t} p(t, x, y) \leq \bar{p}(t, x, y) \leq p(t, x, y)+t\|\bar{J}-J\|_{\infty} \quad \text { for } t>0 \text { and } x, y \in M_{0} .
$$


Since $p(t, x, y)$ satisfies $\operatorname{HK}(\Phi, \psi)$, which is $\operatorname{HK}_{-}\left(\phi_{j}, \phi_{c}\right)$ in the nation of this paper, we have

$$
\bar{p}(t, x, y) \simeq \frac{1}{V\left(x, \phi_{c}^{-1}(t)\right)} \quad \text { for } t \in(0,1] \text { and } d(x, y) \leq \phi_{c}^{-1}(t) .
$$

On the other hand, since

$$
\bar{\phi}_{j}(R) / \bar{\phi}_{j}(r) \leq c_{5}(R / r)^{\bar{\beta}_{2}}
$$

for every $R>r>0$, where $\bar{\beta}_{2}:=\beta_{2} \vee\left(\beta^{*} \wedge(3 / 2)\right)<2$, we have by [CKW1, Remark 1.7 and Theorem 1.13], $\bar{p}(t, x, y)$ enjoys the heat kernel estimate $\operatorname{HK}\left(\bar{\phi}_{j}\right)$. In particular,

$$
\bar{p}(t, x, y) \simeq \frac{1}{V\left(x, \phi_{j}^{-1}(t)\right)} \quad \text { for } t \in(0,1] \text { and } d(x, y) \leq \phi_{j}^{-1}(t)
$$

It follows then from 1.33 that

$$
\frac{1}{V\left(x, \phi_{j}^{-1}(t)\right)} \simeq \frac{1}{V\left(x, \phi_{c}^{-1}(t)\right)} \quad \text { for } t \in(0,1]
$$

This won't be impossible under VD and RVD unless $\beta_{*}>1$, since $\phi_{c}$ is not comparable to $\phi_{j}$ on $(0,1]$ when $\beta_{*} \leq 1$. In summary, BKKL2] essentially requires $\beta_{*} \wedge \beta^{*}>1$.

As mentioned in Remark 1.16, the setting of this paper covers that of [CKW1] as well as BKKL2]. For simplicity, for proofs, we will be mainly concerned with the case that $\phi(r)=$ $\phi_{j}(r) \mathbf{1}_{[0,1]}+\phi_{c}(r) \mathbf{1}_{(1, \infty)}$; that is, the case discussed in Remark 1.17. The other cases can be treated similarly and even easier. On the other hand, as mentioned in Remark 1.17(ii), the expressions of heat kernel estimates for the process studied in this case are the same as these for diffusions with jumps studied in CKW4 but with time interval $t \leq 1$ and $t>1$ switched. So some ideas and strategies from [CKW4 can be used in this case. Indeed, one can follow the arguments in CKW4 to prove Theorems 1.11 and 1.12 in this case, as well as other main assertions, by exchanging the corresponding time intervals. Hence we will skip most of proofs when they are the same, but will illustrate explicitly the differences in the proofs when they are different. We in particular give the proof of the $(2) \Longrightarrow(1)$ part in Theorem 1.12 (see Proposition 3.5 below), which is the most difficult one among all the proofs.

The rest of the paper is organized as follows. In Section 2, we consider the consequences of $\operatorname{UHK}\left(\phi_{j}, \phi_{c}\right)$, and prove the $(1) \Longrightarrow(4)$ part and the $(4) \Longrightarrow(5)$ part of Theorem 1.12 . Section 3 is devoted to proving $\operatorname{UHK}\left(\phi_{j}, \phi_{c}\right)$, which is the most difficult part of the paper. The crucial step is how to use rough tail probability estimates to get $\operatorname{UHK}\left(\phi_{j}, \phi_{c}\right)$. In particular, $(5) \Longrightarrow(3)$, $(3) \Longrightarrow(2)$ and $(2) \Longrightarrow(1)$ in Theorem 1.12 are proven here. With all the results in previous sections, we prove two-sided heat kernel estimates (i.e., $\operatorname{HK}_{-}\left(\phi_{j}, \phi_{c}\right)$ and $\left.\operatorname{HK}\left(\phi_{j}, \phi_{c}\right)\right)$ in Section 4. In Section 5, we also give some applications of heat kernel estimates. In particular, the characterizations of parabolic Harnack inequalities are presented here. Finally, as mentioned earlier, the proof of Example 1.1 is given in the appendix.

Throughout this paper, we will use $c$, with or without subscripts, to denote strictly positive finite constants whose values are insignificant and may change from line to line. For $p \in[1, \infty]$, we will use $\|f\|_{p}$ to denote the $L^{p}$-norm in $L^{p}(M ; \mu)$. For $B=B\left(x_{0}, r\right)$ and $a>0$, we use $a B$ to denote the ball $B\left(x_{0}, a r\right)$. For any subset $D$ of $M, D^{c}:=M \backslash D$. 


\section{Implications of heat kernel estimates}

In this section, we will prove $(1) \Longrightarrow(3)$ and $(3) \Longrightarrow(4)$ in Theorem 1.12 . We first recall the following fact, whose proof is the same as that of [CKW1, Proposition 7.6].

Proposition 2.1. Under VD, RVD and $(1.10)$, we have $\operatorname{UHKD}(\phi) \Longrightarrow \mathrm{FK}(\phi)$ and $\mathrm{PI}(\phi) \Longrightarrow$ $\operatorname{FK}(\phi)$.

2.1 $\operatorname{UHK}\left(\phi_{j}, \phi_{c}\right)+(\mathcal{E}, \mathcal{F})$ is conservative $\Longrightarrow \mathrm{J}_{\phi_{j}, \leq}$ and $\mathrm{HK}_{-}\left(\phi_{j}, \phi_{c}\right) \Longrightarrow \mathrm{J}_{\phi_{j}}$

Proposition 2.2. Under VD and (1.10),

$$
\operatorname{UHK}\left(\phi_{j}, \phi_{c}\right) \text { and }(\mathcal{E}, \mathcal{F}) \text { is conservative } \Longrightarrow \mathrm{J}_{\phi_{j}, \leq}
$$

and

$$
\mathrm{HK}_{-}\left(\phi_{j}, \phi_{c}\right) \Longrightarrow \mathrm{J}_{\phi_{j}}
$$

In particular,

$$
\operatorname{HK}\left(\phi_{j}, \phi_{c}\right) \Longrightarrow \mathrm{J}_{\phi_{j}} \text {. }
$$

Proof. The proof is similar to that of [CKW1, Proposition 3.3] or [CKW4, Proposition 3.1], so we omit it here. We remark that in fact we only need $\operatorname{UHK}\left(\phi_{j}, \phi_{c}\right)\left(\operatorname{resp} . \operatorname{HK}_{-}\left(\phi_{j}, \phi_{c}\right)\right.$ or $\left.\operatorname{HK}\left(\phi_{j}, \phi_{c}\right)\right)$ of $p(t, x, y)$ for sufficiently small $t$.

\section{$2.2 \operatorname{UHK}\left(\phi_{j}, \phi_{c}\right)+(\mathcal{E}, \mathcal{F})$ is conservative $\Longrightarrow \operatorname{Gcap}(\phi)+\operatorname{CSJ}(\phi)$}

In this subsection, we give the proof that $\operatorname{UHK}\left(\phi_{j}, \phi_{c}\right)$ and the conservativeness of $(\mathcal{E}, \mathcal{F})$ imply $\operatorname{Gcap}(\phi)$ and $\operatorname{CSJ}(\phi)$.

The following lemma is frequently used in the proofs of the paper.

Lemma 2.3. ([CKW1, Lemma 2.1]) Assume that VD and 1.10 hold. If $\mathrm{J}_{\phi_{j}, \leq}$ holds, then there exists a constant $c_{1}>0$ such that

$$
\int_{B(x, r)^{c}} J(x, y) \mu(d y) \leq \frac{c_{1}}{\phi_{j}(r)} \quad \text { for every } x \in M \text { and } r>0 .
$$

Similarly, if $\mathrm{J}_{\phi, \leq}$ holds, then there exists a constant $c_{2}>0$ such that

$$
\int_{B(x, r)^{c}} J(x, y) \mu(d y) \leq \frac{c_{2}}{\phi(r)} \quad \text { for every } x \in M \text { and } r>0 .
$$

Proposition 2.4. Assume that $\mathrm{VD}, 1.10$ and $\operatorname{UHK}\left(\phi_{j}, \phi_{c}\right)$ hold and that $(\mathcal{E}, \mathcal{F})$ is conservative. Then $\mathrm{EP}_{\phi, \leq}$ holds. Consequently, $\operatorname{Gcap}(\phi)$ holds.

Proof. According to the proof of [CKW1, Lemma 2.7] and the conservativeness of the process $X$, we only need to verify that there is a constant $c_{1}>0$ such that for each $t, r>0$ and for almost all $x \in M$,

$$
\int_{B(x, r)^{c}} p(t, x, y) \mu(d y) \leq \frac{c_{1} t}{\phi(r)} .
$$

For this, we only need to consider the case that $\phi(r)>t$; otherwise, the inequality above holds trivially with $c_{1}=1$. 
Recall the definition of $\phi(r)$ from (1.14). In the following, we only consider the case that $\phi(r)=\phi_{j}(r) \mathbf{1}_{[0,1]}(r)+\phi_{c}(r) \mathbf{1}_{(1, \infty)}$; other cases can be treated similarly or easily. In this case, $\operatorname{UHK}\left(\phi_{j}, \phi_{c}\right)$ is that for any $x, y \in M_{0}$ and $t>0$,

$$
p(t, x, y) \leq c_{1}^{*} \begin{cases}p^{(j)}(t, x, y), & 0<t \leq 1, \\ \left.\frac{1}{V\left(x, \phi_{c}^{-1}(t)\right)} \wedge\left(p^{(c)}\left(c_{2}^{*} t, x, y\right)+p^{(j)}(t, x, y)\right)\right), & t \geq 1,\end{cases}
$$

where $c_{1}^{*}, c_{2}^{*}>0$ are independent of $x, y \in M_{0}$ and $t>0$.

For $0<t \leq 1$, it follows from the proof of [CKW1, Lemma 2.7] that the desired assertion holds. Hence we consider the case $t \geq 1$. According to the above expression of $\operatorname{UHK}\left(\phi_{j}, \phi_{c}\right)$, VD and (1.10)-1.12), for any $t \geq 1$ and $r \geq 1$ with $\phi_{c}(r)>t$ and every $x \in M_{0}$,

$$
\begin{aligned}
& \int_{B(x, r)^{c}} p(t, x, y) \mu(d y) \\
& =\sum_{i=0}^{\infty} \int_{B\left(x, 2^{i+1} r\right) \backslash B\left(x, 2^{i} r\right)} p(t, x, y) \mu(d y) \\
& \leq \sum_{i=0}^{\infty} \frac{c_{2} V\left(x, 2^{i+1} r\right)}{V\left(x, \phi_{c}^{-1}(t)\right)} \exp \left(-\frac{c_{3} 2^{i} r}{\bar{\phi}_{c}^{-1}\left(t /\left(2^{i} r\right)\right)}\right)+\sum_{i=0}^{\infty} \frac{c_{2} t V\left(x, 2^{i+1} r\right)}{V\left(x, 2^{i} r\right) \phi_{j}\left(2^{i} r\right)} \\
& \leq c_{4} \sum_{i=0}^{\infty}\left(\frac{2^{i} r}{\phi_{c}^{-1}(t)}\right)^{d_{2}} \exp \left(-\frac{c_{5} 2^{i} r}{\bar{\phi}_{c}^{-1}(t / r)}\right)+\frac{c_{4} t}{\phi_{j}(r)} \sum_{i=0}^{\infty} 2^{-i \beta_{1, \phi_{j}}} \\
& \leq c_{6} \sum_{i=0}^{\infty}\left(2^{i}\left(\frac{\phi_{c}(r)}{t}\right)^{1 / \beta_{1, \phi_{c}}}\right)^{d_{2}} \exp \left(-c_{7} 2^{i}\left(\frac{\phi_{c}(r)}{t}\right)^{1 /\left(\beta_{2, \phi_{c}}-1\right)}\right)+\frac{c_{6} t}{\phi_{j}(r)} \\
& \leq c_{6} \sum_{i=0}^{\infty}\left(2^{i\left(\beta_{2, \phi_{c}}-1\right)} \frac{\phi_{c}(r)}{t}\right)^{d_{2}\left(1+1 /\left(\beta_{2, \phi_{c}}-1\right)\right)} \exp \left(-c_{7}\left(2^{i\left(\beta_{2, \phi_{c}}-1\right)} \frac{\phi_{c}(r)}{t}\right)^{1 /\left(\beta_{2, \phi_{c}}-1\right)}\right)+\frac{c_{6} t}{\phi_{j}(r)} \\
& \leq c_{8} \sum_{i=0}^{\infty} \exp \left(-\frac{c_{7}}{2}\left(2^{i\left(\beta_{2, \phi_{c}}-1\right)} \frac{\phi_{c}(r)}{t}\right)^{1 /\left(\beta_{2, \phi_{c}}-1\right)}\right)+\frac{c_{6} t}{\phi_{j}(r)} \\
& \leq c_{9} t\left(\frac{1}{\phi_{c}(r)}+\frac{1}{\phi_{j}(r)}\right) \leq \frac{c_{10} t}{\phi(r)},
\end{aligned}
$$

where in the second inequality we used the fact that $\bar{\phi}_{c}(r)$ is increasing on $(0, \infty)$, the fourth inequality we used the fact that $\beta_{1, \phi_{c}}>1$, in the fifth and the sixth inequalities we used the facts that for there is a constant $c_{11}>0$ such that

$$
s^{d_{2}\left(1+1 /\left(\beta_{2, \phi_{c}}-1\right)\right)} \leq c_{11} \exp \left(\frac{c_{7}}{2} s^{1 /\left(\beta_{2, \phi_{c}}-1\right)}\right), \quad s \geq 1
$$

and

$$
\sum_{i=0}^{\infty} \exp \left(-\frac{c_{7}}{2}\left(2^{i\left(\beta_{2, \phi_{c}}-1\right)} s\right)^{1 /\left(\beta_{2, \phi_{c}}-1\right)}\right) \leq c_{11} / s, \quad s \geq 1,
$$

respectively, and the last inequality is due to 1.13$)$. The proof of $\mathrm{EP}_{\phi, \leq, \varepsilon} \Longrightarrow \operatorname{Gcap}(\phi)$ is the same as that of [GHH, Lemma 2.8], 
Proposition 2.5. Under VD and 1.10$), \operatorname{Gcap}(\phi)$ and $\mathrm{J}_{\phi, \leq}$ imply $\operatorname{CSJ}(\phi)$. In particular, suppose that $\mathrm{VD}, 1.10$ and $\operatorname{UHK}\left(\phi_{j}, \phi_{c}\right)$ hold, and that $(\mathcal{E}, \mathcal{F})$ is conservative. Then $\operatorname{CSJ}(\phi)$ holds.

Proof. The first assertion follows from that of [GHH, Lemma 2.4] (or that of [CKW4, Proposition 2.5]). This along with Proposition 2.2 and Lemma 2.4 gives us the second one. Indeed, we also can follow the argument of [CKW1, Proposition 3.6] to verify the second assertion directly.

\section{Implications of $\mathrm{FK}(\phi), \operatorname{CSJ}(\phi)$ and $\mathrm{J}_{\phi_{j}, \leq}\left(\right.$ or $\left.\mathrm{J}_{\phi, \leq}\right)$}

\section{$3.1 \mathrm{FK}(\phi)+\mathrm{J}_{\phi, \leq}+\operatorname{CSJ}(\phi) \Rightarrow \mathrm{E}_{\phi}$, and $\mathrm{FK}(\phi)+\mathrm{J}_{\phi, \leq}+\mathrm{E}_{\phi} \Rightarrow \operatorname{UHKD}(\phi)$}

The next two propositions can be proved by following arguments in [CKW1].

Proposition 3.1. Assume VD, 1.10, $\mathrm{FK}(\phi), \mathrm{J}_{\phi, \leq}$ and $\operatorname{CSJ}(\phi)$ hold. Then $\mathrm{E}_{\phi}$ holds.

Proof. According to [CKW1, Lemma 4.14], $\mathrm{E}_{\phi, \leq}$ holds under VD, 1.10) and $\mathrm{FK}(\phi)$. On the other hand, following the arguments of [CKW1, Lemmas 4.15, 4.16 and 4.17], we can obtain $\mathrm{E}_{\phi, \geq}$. Here, we note that under assumptions of proposition, one can obtain mean value inequalities in the present setting as those in [CKW1, Proposition 4.11 and Corollary 4.12], and so the arguments above go through.

Proposition 3.2. Suppose that VD, 1.10$), \mathrm{FK}(\phi), \mathrm{E}_{\phi}$ and $\mathrm{J}_{\phi, \leq}$ hold. Then $\mathrm{UHKD}(\phi)$ is satisfied, i.e., there is a constant $c>0$ such that for all $x \in M_{0}$ and $t>0$,

$$
p(t, x, x) \leq \frac{c}{V\left(x, \phi^{-1}(t)\right)} .
$$

Proof. The proof is the same as that of [CKW1, Theorem 4.25], and so we omit it here.

Propositions 3.1 and 3.2 give the $(5) \Longrightarrow(3)$ part and the $(3) \Longrightarrow(2)$ part of Theorem 1.12

\section{$3.2 \operatorname{UHKD}(\phi)+\mathrm{J}_{\phi_{j}, \leq}+\mathrm{E}_{\phi} \Rightarrow \operatorname{UHK}\left(\phi_{j}, \phi_{c}\right)$}

In this part, we will prove $(2) \Longrightarrow(1)$ in Theorem 1.12 . For this, we need to use the truncation technique. Fix $\rho>0$ and define a bilinear form $\left(\mathcal{E}^{(\rho)}, \mathcal{F}\right)$ by

$$
\mathcal{E}^{(\rho)}(u, v)=\int(u(x)-u(y))(v(x)-v(y)) \mathbf{1}_{\{d(x, y) \leq \rho\}} J(d x, d y) .
$$

Clearly, the form $\mathcal{E}^{(\rho)}(u, v)$ is well defined for $u, v \in \mathcal{F}$, and $\mathcal{E}^{(\rho)}(u, u) \leq \mathcal{E}(u, u)$ for all $u \in \mathcal{F}$. Assume that VD, 1.10 and $\mathrm{J}_{\phi, \leq}$ hold. Then we have by Lemma 2.3 that for all $u \in \mathcal{F}$,

$$
\begin{aligned}
\mathcal{E}(u, u)-\mathcal{E}^{(\rho)}(u, u) & =\int(u(x)-u(y))^{2} \mathbf{1}_{\{d(x, y)>\rho\}} J(d x, d y) \\
& \leq 4 \int_{M} u^{2}(x) \mu(d x) \int_{B(x, \rho)^{c}} J(x, y) \mu(d y) \leq \frac{c_{0}\|u\|_{2}^{2}}{\phi(\rho)} .
\end{aligned}
$$


Thus, $\mathcal{E}_{1}(u, u)$ is equivalent to $\mathcal{E}_{1}^{(\rho)}(u, u):=\mathcal{E}^{(\rho)}(u, u)+\|u\|_{2}^{2}$ for every $u \in \mathcal{F}$. Hence $\left(\mathcal{E}^{(\rho)}, \mathcal{F}\right)$ is a regular Dirichlet form on $L^{2}(M ; \mu)$. Throughout this paper, we call $\left(\mathcal{E}^{(\rho)}, \mathcal{F}\right)$ the $\rho$-truncated Dirichlet form. The Hunt process associated with $\left(\mathcal{E}^{(\rho)}, \mathcal{F}\right)$, denoted by $\left(X_{t}^{(\rho)}\right)_{t \geq 0}$, can be identified in distribution with the Hunt process of the original Dirichlet form $(\mathcal{E}, \mathcal{F})$ by removing those jumps of size larger than $\rho$.

According to the proof of [CKW1, Lemma 5.2], we have the following statement.

Lemma 3.3. Assume that $\mathrm{VD},(1.10), \operatorname{UHKD}(\phi), \mathrm{J}_{\phi, \leq}$ and $\mathrm{E}_{\phi}$ hold. Then the $\rho$-truncated Dirichlet form $\left(\mathcal{E}^{(\rho)}, \mathcal{F}\right)$ has the heat kernel $q^{(\rho)}(t, x, y)$, and it holds that for any $t>0$ and all $x, y \in M_{0}$,

$$
q^{(\rho)}(t, x, y) \leq c_{1}\left(\frac{1}{V\left(x, \phi^{-1}(t)\right)}+\frac{1}{V\left(y, \phi^{-1}(t)\right)}\right) \exp \left(c_{2} \frac{t}{\phi(\rho)}-c_{3} \frac{d(x, y)}{\rho}\right),
$$

where $c_{1}, c_{2}, c_{3}$ are positive constants independent of $\rho$.

Consequently, the following holds for any $t>0$ and all $x, y \in M_{0}$,

$$
q^{(\rho)}(t, x, y) \leq \frac{c_{4}}{V\left(x, \phi^{-1}(t)\right)}\left(1+\frac{d(x, y)}{\phi^{-1}(t)}\right)^{d_{2}} \exp \left(c_{2} \frac{t}{\phi(\rho)}-c_{3} \frac{d(x, y)}{\rho}\right) .
$$

From here to the end of this section, we will assume $\mathrm{J}_{\phi_{j}, \leq}$ instead of $\mathrm{J}_{\phi, \leq}$. First, the relation between $p(t, x, y)$ and $q^{(\rho)}(t, x, y)$ can be seen through the Meyer's decomposition; e.g. see [CKW1, Section 7.2]. In particular, according to [CKW1, (4.34) and Proposition 4.24 (and its proof)], for any $t, \rho>0$ and all $x, y \in M_{0}$,

$$
p(t, x, y) \leq q^{(\rho)}(t, x, y)+\frac{c_{1} t}{V(x, \rho) \phi_{j}(\rho)} \exp \left(\frac{c_{1} t}{\phi(\rho)}\right),
$$

where $c_{1}$ is independent of $t, \rho>0$ and $x, y \in M_{0}$. With this at hand, in order to deduce full upper bounds for the heat kernel, we will make use of tail estimates of the transition probability. Namely, we will use the following lemma, which is essentially taken from [BKKL1, Lemma 4.3] and partly motivated by the proof of [CKW1, Proposition 5.3].

Lemma 3.4. Assume that $\mathrm{VD}, 1.10), \operatorname{UHKD}(\phi), \mathrm{J}_{\phi_{j}, \leq}$ and $\mathrm{E}_{\phi}$ hold. Let $f: \mathbb{R}_{+} \times \mathbb{R}_{+} \rightarrow \mathbb{R}_{+}$ be a measurable function satisfying that $t \mapsto f(r, t)$ is non-increasing for all $r>0$, and that $r \mapsto f(r, t)$ is non-decreasing for all $t>0$. Suppose that the following hold:

(i) For each $b>0, \sup _{t>0} f\left(b \phi^{-1}(t), t\right)<\infty$.

(ii) There exist constants $\eta \in\left(0, \beta_{1, \phi_{j}}\right]$ and $a_{1}, c_{1}>0$ such that for all $x \in M_{0}$ and $r, t>0$,

$$
\int_{B(x, r)^{c}} p(t, x, y) \mu(d y) \leq c_{1}\left(\frac{\phi_{j}^{-1}(t)}{r}\right)^{\eta}+c_{1} \exp \left(-a_{1} f(r, t)\right) .
$$

Then, there exist constants $k, c_{0}>0$ such that for all $x, y \in M_{0}$ and $t>0$,

$$
\begin{aligned}
p(t, x, y) \leq & \frac{c_{0} t}{V(x, d(x, y)) \phi_{j}(d(x, y))} \\
& +\frac{c_{0}}{V\left(x, \phi^{-1}(t)\right)}\left(1+\frac{d(x, y)}{\phi^{-1}(t)}\right)^{d_{2}} \exp \left(-a_{1} k f(d(x, y) /(16 k), t)\right) .
\end{aligned}
$$

Furthermore, the conclusion still holds true for any $t \in(0, T]$ or $t \in[T, \infty)$ with some $T>0$, if assumptions (i) and (ii) above are restricted on the corresponding time interval. 
Proof. The proof is the same as that of [CKW4, Lemma 4.5] so it is omitted here.

Proposition 3.5. Suppose $\mathrm{VD}$ and 1.10 , $\operatorname{UHKD}(\phi), \mathrm{J}_{\phi_{j}, \leq}$ and $\mathrm{E}_{\phi}$ hold. Then we have $\operatorname{UHK}\left(\phi_{j}, \phi_{c}\right)$.

While the idea of the proof of Proposition 3.5 is similar to that of [CKW4, Proposition 4.6], the actual proof requires lots of modifications. To illustrate the differences of the proofs between CKW4 and the present setting, below we give the proof of Proposition 3.5 in details. For the proof, we need the following two lemmas in addition to Lemma 3.4 .

Lemma 3.6. Suppose $\mathrm{VD}$ and $1.10, \operatorname{UHKD}(\phi), \mathrm{J}_{\phi_{j}, \leq}$ and $\mathrm{E}_{\phi}$ hold. Then there exist constants $a, c>0$ and $N \in \mathbb{N}$ such that for all $x, y \in M_{0}$ and $t>0$,

$$
p(t, x, y) \leq \frac{c t}{V(x, d(x, y)) \phi_{j}(d(x, y))}+\frac{c}{V\left(x, \phi^{-1}(t)\right)} \exp \left(-\frac{a d(x, y)^{1 / N}}{\phi^{-1}(t)^{1 / N}}\right) .
$$

Proof. The proof is the same as that of [CKW4, Lemma 4.7].

Lemma 3.7. For any $C_{*}>0$, there exist constants $C_{0}, C^{*}>0$ such that

(i) for any $t \in[1, \infty)$ and $r \geq C_{0} \phi_{c}^{-1}(t)$, the function

$$
r \mapsto F_{1, t}(r):=\frac{\exp \left(C_{*} r / \bar{\phi}_{c}^{-1}(t / r)\right)}{r / \bar{\phi}_{c}^{-1}(t / r)}
$$

is strictly increasing.

(ii) for any $t \in[1, \infty)$, there is a unique $r_{*}(t) \in\left(C_{0} \phi_{c}^{-1}(t), \infty\right)$ such that $F_{1, t}\left(r_{*}(t)\right)=$ $F_{2, t}\left(r_{*}(t)\right)$

$$
F_{1, t}(r)<F_{2, t}(r), \quad r \in\left(C_{0} \phi_{c}^{-1}(t), r_{*}(t)\right)
$$

and

$$
F_{1, t}(r)>F_{2, t}(r), \quad r \in\left(r_{*}(t), \infty\right),
$$

where

$$
F_{2, t}(r)=\frac{C^{*} \bar{\phi}_{c}^{-1}(t / r)}{\phi_{j}^{-1}(t)}
$$

Proof. (i) We know from 11.10 and 1.12 that, if $r \geq C_{0} \phi_{c}^{-1}(t)$ with $C_{0}$ large enough, then

$$
\frac{r}{\bar{\phi}_{c}^{-1}(t / r)} \geq c_{1}\left(\frac{\phi_{c}(r)}{t}\right)^{1 /\left(\beta_{2, \phi_{c}}-1\right)} \geq c_{2}\left(\frac{r}{\phi_{c}^{-1}(t)}\right)^{\beta_{1, \phi_{c}} /\left(\beta_{2, \phi_{c}}-1\right)} \geq c_{2} C_{0}^{\beta_{1, \phi_{c}} /\left(\beta_{2, \phi_{c}}-1\right)},
$$

where the constants $c_{1}$ and $c_{2}$ are independent of $C_{0}$. Note further that the function $r \mapsto$ $r / \bar{\phi}_{c}^{-1}(t / r)$ is strictly increasing due to the strictly increasing property of the function $\bar{\phi}_{c}(r)$ on $\mathbb{R}_{+}$. Then, the first required assertion follows from the fact that $s \mapsto e^{C_{*} s} / s$ is strictly increasing on $\left[c_{2} C_{0}^{\beta_{1, \phi_{c}} /\left(\beta_{2, \phi_{c}}-1\right)}, \infty\right)$, by choosing $C_{0}$ (depending on $C_{*}$ ) large enough.

(ii) As seen from (i) and its proof that the function $F_{1, t}(r)$ is strictly increasing on $\left[C_{0} \phi_{c}^{-1}(t), \infty\right)$ with $F_{1, t}(\infty)=\infty$. On the other hand, due to the strictly increasing property of the function 
$\bar{\phi}_{c}(r)$ on $\mathbb{R}_{+}$and $(1.12)$, we know that the function $F_{2, t}(r)$ is strictly decreasing on $\left[C_{0} \phi_{c}^{-1}(t), \infty\right)$ with $F_{2, t}\left(C_{0} \phi_{c}^{-1}(t)\right)=C^{*} \bar{\phi}_{c}^{-1}\left(t /\left(C_{0} \phi_{c}^{-1}(t)\right)\right) / \phi_{j}^{-1}(t)$ and $F_{2, t}(\infty)=0$.

Furthermore, according to 1.10 and 1.12 again, and the fact that $\phi_{c}^{-1}(t) \geq \phi_{j}^{-1}(t)$ for all $t \in[1, \infty)$, we can obtain that

$$
F_{1, t}\left(C_{0} \phi_{c}^{-1}(t)\right) \leq c_{3}
$$

and

$$
F_{2, t}\left(C_{0} \phi_{c}^{-1}(t)\right)=\frac{C^{*} \bar{\phi}_{c}^{-1}\left(t /\left(C_{0} \phi_{c}^{-1}(t)\right)\right)}{\phi_{c}^{-1}(t)} \frac{\phi_{c}^{-1}(t)}{\phi_{j}^{-1}(t)} \geq \frac{C^{*} \bar{\phi}_{c}^{-1}\left(t /\left(C_{0} \phi_{c}^{-1}(t)\right)\right)}{\phi_{c}^{-1}(t)} \geq C^{*} c_{4},
$$

where $c_{3}, c_{4}$ are independent of $C^{*}$.

Combining with both conclusions above and taking $C^{*}=2 c_{3} / c_{4}$, we then prove the second desired assertion.

Proof of Proposition 3.5. We only give the proof for the case of $\beta_{*} \leq 1$. The proof for the case of $\beta_{*}>1$ is quite similar.

Let $N \geq 1$ be the positive integer in Lemma 3.6. We have by VD and 1.15 that for $k \geq 1$, there are positive constants $c_{2}=c_{2}(k)$ and $c_{3}=c_{3}(k)$ so that any $t>0$ and $x, y \in M_{0}$ with $d(x, y) \geq \phi^{-1}(t)$

$$
\begin{aligned}
\frac{1}{V\left(x, \phi^{-1}(t)\right)} \exp \left(-\frac{a d(x, y)^{1 / N}}{\phi^{-1}(t)^{1 / N}}\right) & \leq \frac{c_{1}}{V(x, d(x, y))}\left(\frac{d(x, y)}{\phi^{-1}(t)}\right)^{d_{2}} \exp \left(-\frac{a d(x, y)^{1 / N}}{\phi^{-1}(t)^{1 / N}}\right) \\
& \leq \frac{c_{2}}{V(x, d(x, y))}\left(\frac{\phi^{-1}(t)}{d(x, y)}\right)^{k \beta_{2, \phi}} \\
& \leq \frac{c_{3} t^{k}}{V(x, d(x, y)) \phi(d(x, y))^{k}}
\end{aligned}
$$

where in the second inequality we used the fact that $r^{d_{2}+k \beta_{2, \phi}} e^{-a r^{1 / N} / 2} \leq c_{4}(k)$ for every $r \geq 1$.

Suppose first that $\beta^{*} \leq 1$. Then $\phi(r)=\phi_{j}(r)$ for all $r>0$. By Lemma 3.6, there exist constants $a, c>0$ and $N \in \mathbb{N}$ such that for all $x, y \in M_{0}$ and $t>0$,

$$
p(t, x, y) \leq \frac{c t}{V(x, d(x, y)) \phi_{j}(d(x, y))}+\frac{c}{V\left(x, \phi_{j}^{-1}(t)\right)} \exp \left(-\frac{a d(x, y)^{1 / N}}{\phi_{j}^{-1}(t)^{1 / N}}\right) .
$$

Hence we have by $(3.2)$ with $k=1$ and $\operatorname{UHKD}(\phi)$ that for every $x, y \in M_{0}$ and $t>0$,

$$
p(t, x, y) \leq c_{5}\left(\frac{1}{V\left(x, \phi_{j}^{-1}(t)\right)} \wedge \frac{t}{V(x, d(x, y)) \phi_{j}(d(x, y))}\right)
$$

which proves $\operatorname{UHK}\left(\phi_{j}, \phi_{c}\right)$ (i.e., $\operatorname{UHK}\left(\phi_{j}\right)$ in [CKW1] ).

Now we consider the case of $\beta^{*}>1$. We split it into two cases.

(1) Case: $0<t \leq 1$. Since $\beta_{*} \leq 1, \phi(r)=\phi_{j}(r)$ for $r \in[0,1]$. By (1.10) and (1.15), there is some $k_{0} \geq 1$ so that $\phi(r)^{k_{0}} \geq c_{6} \phi_{j}(r)$ on $[1, \infty)$. We have by Lemma 3.6 and $(3.2)$ with $k=k_{0}$ when $d(x, y) \geq 1$ and with $k=1$ when $1 \geq d(x, y) \geq \phi^{-1}(t)=\phi_{j}^{-1}(t)$ that for all $t \in(0,1]$ and $x, y \in M_{0}$ with $d(x, y) \geq \phi_{j}^{-1}(t)$,

$$
p(t, x, y) \leq \frac{c_{6} t}{V(x, d(x, y)) \phi_{j}(d(x, y))},
$$


This together with $\operatorname{UHKD}(\phi)$ establishes $\operatorname{UHK}\left(\phi_{j}, \phi_{c}\right)$ when $0<t \leq 1$.

(2) Case: $t \geq 1$. By $\operatorname{UHKD}(\phi)$, it suffices to consider the case that $x, y \in M_{0}$ and $t \geq 1$ with $d(x, y) \geq \phi^{-1}(t)=\phi_{c}^{-1}(t)$. According to Lemma 3.4 it is enough to verify that there exist constants $\eta \in\left(0, \beta_{1, \phi_{j}}\right]$ and $c_{1}, c_{2}>0$ such that for any $x \in M_{0}, t \geq 1$ and $r>0$,

$$
\int_{B(x, r)^{c}} p(t, x, y) \mu(d y) \leq c_{1}\left(\frac{\phi_{j}^{-1}(t)}{r}\right)^{\eta}+c_{1} \exp \left(-c_{2} \frac{r}{\bar{\phi}_{c}^{-1}(t / r)}\right) .
$$

Indeed, by 3.3 , Lemma 3.4 with $f(r, t)=r / \bar{\phi}_{c}^{-1}(t / r)$, we have that for any $x, y \in M_{0}$ and $t \geq 1$,

$$
p(t, x, y) \leq c_{3}\left(\frac{t}{V(x, d(x, y)) \phi_{j}(d(x, y))}+\frac{1}{V\left(x, \phi_{c}^{-1}(t)\right)} \exp \left(-\frac{c_{0}^{*} d(x, y)}{\bar{\phi}_{c}^{-1}(t / d(x, y))}\right)\right) .
$$

Therefore, combining both conclusions above, we can obtain the desired assertion. In the following, we will prove (3.3). For this, we will consider four different cases.

(i) If $r \leq C_{0} \phi_{c}^{-1}(t)$ for some $C_{0}>0$ which is determined in the step (ii) below, then, by the non-decreasing property of $\bar{\phi}_{c}, 1.11$ and 1.12 ,

$$
\exp \left(c_{2} \frac{r}{\bar{\phi}_{c}^{-1}(t / r)}\right) \leq \exp \left(c_{2} \frac{C_{0} \phi_{c}^{-1}(t)}{\bar{\phi}_{c}^{-1}\left(t /\left(C_{0} \phi_{c}^{-1}(t)\right)\right)}\right) \leq e^{c_{4}}
$$

Hence, (3.3) holds trivially by taking $c_{1}=e^{c_{4}}$.

(ii) Suppose that $C_{0} \phi_{c}^{-1}(t) \leq r \leq r_{*}(t)$, where $r_{*}(t)$ is determined later. For any $n \in \mathbb{N} \cup\{0\}$, define

$$
\rho_{n}=c_{*} 2^{n \alpha} \bar{\phi}_{c}^{-1}\left(\frac{t}{r}\right)
$$

with $d_{2} /\left(d_{2}+\beta_{1, \phi_{j}}\right)<\alpha<1$, where $c_{*}$ is also determined later. Then, by (3.1) and Lemma 3.3. for any $x, y \in M_{0}$ with $2^{n} r \leq d(x, y) \leq 2^{n+1} r$ and $t \geq 1$,

$$
\begin{aligned}
p(t, x, y) \leq & \frac{c_{1}}{V\left(x, \phi_{c}^{-1}(t)\right)}\left(1+\frac{2^{n+1} r}{\phi_{c}^{-1}(t)}\right)^{d_{2}} \exp \left(\frac{c_{1} t}{\phi\left(\rho_{n}\right)}-\frac{c_{2} 2^{n} r}{\rho_{n}}\right) \\
& +\frac{c_{1} t}{V\left(x, \rho_{n}\right) \phi_{j}\left(\rho_{n}\right)} \exp \left(\frac{c_{1} t}{\phi\left(\rho_{n}\right)}\right) \\
\leq & \frac{c_{1}}{V\left(x, \phi_{c}^{-1}(t)\right)}\left(1+\frac{2^{n+1} r}{\phi_{c}^{-1}(t)}\right)^{d_{2}} \exp \left(\frac{c_{1} t}{\phi_{c}\left(\rho_{n}\right)}-\frac{c_{2} 2^{n} r}{\rho_{n}}\right) \\
& +\frac{c_{1} t}{V\left(x, \rho_{n}\right) \phi_{j}\left(\rho_{n}\right)} \exp \left(\frac{c_{1} t}{\phi_{c}\left(\rho_{n}\right)}\right)
\end{aligned}
$$

where in the last inequality we used the facts that if $0<\rho_{n} \leq 1$, then $\phi\left(\rho_{n}\right)=\phi_{j}\left(\rho_{n}\right) \geq \phi_{c}\left(\rho_{n}\right)$, and if $\rho_{n} \geq 1$, then $\phi\left(\rho_{n}\right)=\phi_{c}\left(\rho_{n}\right)$. Hence, for any $x \in M_{0}, t \geq 1$ and $C_{0} \phi_{c}^{-1}(t) \leq r \leq r_{*}(t)$,

$$
\begin{aligned}
& \int_{B(x, r)^{c}} p(t, x, y) \mu(d y) \\
& =\sum_{n=0}^{\infty} \int_{B\left(x, 2^{n+1} r\right) \backslash B\left(x, 2^{n} r\right)} p(t, x, y) \mu(d y)
\end{aligned}
$$




$$
\begin{aligned}
\leq & \sum_{n=0}^{\infty} \frac{c_{3} V\left(x, 2^{n+1} r\right)}{V\left(x, \phi_{c}^{-1}(t)\right)}\left(1+\frac{2^{n+1} r}{\phi_{c}^{-1}(t)}\right)^{d_{2}} \exp \left(\frac{c_{5} t}{c_{*}^{\beta_{1, \phi_{c}}} 2^{n \alpha \beta_{1, \phi_{c}}} \phi_{c}\left(\bar{\phi}_{c}^{-1}(t / r)\right)}-\frac{c_{2} 2^{n(1-\alpha)} r}{c_{*} \bar{\phi}_{c}^{-1}(t / r)}\right) \\
& +\sum_{n=0}^{\infty} \frac{c_{4} t V\left(x, 2^{n+1} r\right)}{V\left(x, \rho_{n}\right) \phi_{j}\left(\rho_{n}\right)} \exp \left(\frac{c_{5} t}{c_{*}^{\beta_{1, \phi_{c}}} 2^{n \alpha \beta_{1, \phi_{c}}} \phi_{c}\left(\bar{\phi}_{c}^{-1}(t / r)\right)}\right) \\
= & : I_{1}+I_{2},
\end{aligned}
$$

where the inequality above follows from 1.10 , and all the constants above are independent of $c_{*}$. On the one hand, by taking $c_{*}=\left(1+\left(2 c_{1} c_{5} / c_{2}\right)\right)^{1 /\left(\beta_{1, \phi_{c}}-1\right)}$ (with $c_{1} \geq 1$ being the constant in (1.11), we get by VD and (1.11) that

$$
I_{1} \leq c_{6} \sum_{n=0}^{\infty}\left(\frac{2^{n} r}{\phi_{c}^{-1}(t)}\right)^{2 d_{2}} \exp \left(-c_{7} 2^{n(1-\alpha)} \frac{r}{\bar{\phi}_{c}^{-1}(t / r)}\right) \leq c_{8} \exp \left(-c_{9} \frac{r}{\bar{\phi}_{c}^{-1}(t / r)}\right) .
$$

Here and in what follows, the constants will depend on $c_{*}$. On the other hand, according to VD, (1.10) and 1.11 again,

$$
\begin{aligned}
I_{2} & \leq c_{10} \exp \left(\frac{c_{11} r}{\bar{\phi}_{c}^{-1}(t / r)}\right) \sum_{n=0}^{\infty}\left(\frac{2^{n} r}{\rho_{n}}\right)^{d_{2}} \frac{\phi_{j}(r)}{\phi_{j}\left(\rho_{n}\right)} \frac{t}{\phi_{j}(r)} \\
& \leq c_{12} \exp \left(\frac{c_{11} r}{\bar{\phi}_{c}^{-1}(t / r)}\right)\left(\frac{r}{\bar{\phi}_{c}^{-1}(t / r)}\right)^{d_{2}+\beta_{2, \phi_{j}}} \frac{t}{\phi_{j}(r)} \sum_{n=0}^{\infty} 2^{n\left(d_{2}-\alpha\left(d_{2}+\beta_{1, \phi_{j}}\right)\right)} \\
& \leq c_{13} \exp \left(\frac{c_{14} r}{\bar{\phi}_{c}^{-1}(t / r)}\right) \frac{t}{\phi_{j}(r)}
\end{aligned}
$$

where in the last inequality we used the condition $d_{2} /\left(d_{2}+\beta_{1, \phi_{j}}\right)<\alpha<1$ and the fact that

$$
r^{d_{2}+\beta_{2, \phi_{j}}} \leq c_{15} e^{r} \quad \text { for all } r \geq c_{16}>0 .
$$

We emphasize that the argument up to here is independent of the definition of $r_{*}(t)$ and the choice of the constant $C_{0}$. In view of Lemma 3.7, we can find constants $C_{0}, c_{17}>0$ such that

$$
\begin{gathered}
\exp \left(\frac{2 c_{14} r}{\beta_{1, \phi_{j}} \bar{\phi}_{c}^{-1}(t / r)}\right) \leq \frac{c_{17} r}{\phi_{j}^{-1}(t)} \quad \text { when } C_{0} \phi_{c}^{-1}(t) \leq r \leq r_{*}(t), \\
\exp \left(\frac{2 c_{14} r}{\beta_{1, \phi_{j}} \bar{\phi}_{c}^{-1}(t / r)}\right) \geq \frac{c_{17} r}{\phi_{j}^{-1}(t)} \quad \text { for } r \geq r_{*}(t)
\end{gathered}
$$

and

$$
\exp \left(\frac{2 c_{14} r_{*}(t)}{\beta_{1, \phi_{j}} \bar{\phi}_{c}^{-1}\left(t / r_{*}(t)\right)}\right)=\frac{c_{17} r_{*}(t)}{\phi_{j}^{-1}(t)} .
$$

(Here, we note that, without loss of generality we may and do assume that $\frac{2 c_{14}}{\beta_{1, \phi_{j}}} \geq 1$.) Then, due to 1.10 and 3.4 ,

$$
I_{2} \leq c_{18}\left(\frac{r}{\phi_{j}^{-1}(t)}\right)^{\beta_{1, \phi_{j}} / 2}\left(\frac{t}{\phi_{j}(r)}\right) \leq c_{19}\left(\frac{\phi_{j}^{-1}(t)}{r}\right)^{\beta_{1, \phi_{j}} / 2} .
$$


Putting $I_{1}$ and $I_{2}$ together, we arrive at $(3.3)$.

For the need in steps (iii) and (iv) later, we will consider bounds for $r_{*}(t)$. We first treat the lower bound for $r_{*}(t)$. Let $r \geq C_{0} \phi_{c}^{-1}(t)$. By 1.10 and $(1.12)$, we have

$$
\exp \left(\frac{2 c_{14} r}{\beta_{1, \phi_{j}} \bar{\phi}_{c}^{-1}(t / r)}\right) \leq \exp \left(c_{20}\left(\frac{r}{\phi_{c}^{-1}(t)}\right)^{\beta_{2, \phi_{c}} /\left(\beta_{1, \phi_{c}}-1\right)}\right) .
$$

Hence, 3.4 holds, if

$$
\exp \left(c_{20}\left(\frac{r}{\phi_{c}^{-1}(t)}\right)^{\beta_{2, \phi_{c}} /\left(\beta_{1, \phi_{c}}-1\right)}\right) \leq \frac{c_{17} r}{\phi_{j}^{-1}(t)}, \quad C_{0} \phi_{c}^{-1}(t) \leq r \leq r_{*}(t) ;
$$

namely,

$$
\log c_{17}+\log \frac{r}{\phi_{c}^{-1}(t)}+\log \frac{\phi_{c}^{-1}(t)}{\phi_{j}^{-1}(t)} \geq c_{20}\left(\frac{r}{\phi_{c}^{-1}(t)}\right)^{\beta_{2, \phi_{c}} /\left(\beta_{1, \phi_{c}}-1\right)}
$$

holds for all $C_{0} \phi_{c}^{-1}(t) \leq r \leq r_{*}(t)$. From this, one can see that

$$
r_{*}(t) \geq C_{1} \phi_{c}^{-1}(t) \log \left(\beta_{\left.1, \phi_{c}-1\right) / \beta_{2, \phi_{c}}}\left(\phi_{c}^{-1}(t) / \phi_{j}^{-1}(t)\right)\right.
$$

for some constant $C_{1}>0$ which is independent of $t$. For the upper bound for $r_{*}(t)$, similar to the argument for (3.6), we have

$$
\exp \left(\frac{2 c_{14} r}{\beta_{1, \phi_{j}} \bar{\phi}_{c}^{-1}(t / r)}\right) \geq \exp \left(c_{21}\left(\frac{r}{\phi_{c}^{-1}(t)}\right)^{\beta_{1, \phi_{c}} /\left(\beta_{2, \phi_{c}}-1\right)}\right) .
$$

Hence, we can find that

$$
r_{*}(t) \leq C_{2} \phi_{c}^{-1}(t) \log \left(\beta_{2, \phi_{c}}-1\right) / \beta_{1, \phi_{c}}\left(\phi_{c}^{-1}(t) / \phi_{j}^{-1}(t)\right)
$$

where $C_{2}>0$ is also independent of $t$.

(iii) Suppose that $r \geq r^{*}(t):=C_{3} \phi_{c}^{-1}(t) \log ^{N}\left(\phi_{c}^{-1}(t) / \phi_{j}^{-1}(t)\right)$, where $N \in \mathbb{N}$ is given in Lemma 3.6, and $C_{3}>0$ is determined later. According to Lemma 3.6, there exist constants $a, c>0$ and $N \in \mathbb{N}$ such that for all $x, y \in M_{0}$ and $t \geq 1$ with $d(x, y) \geq r^{*}(t)$,

$$
\begin{aligned}
p(t, x, y) & \leq \frac{c t}{V(x, d(x, y)) \phi_{j}(d(x, y))}+\frac{c}{V\left(x, \phi_{c}^{-1}(t)\right)} \exp \left(-\frac{a d(x, y)^{1 / N}}{\phi_{c}^{-1}(t)^{1 / N}}\right) \\
& \leq \frac{c t}{V(x, d(x, y)) \phi_{j}(d(x, y))}+\frac{c_{1}}{V(x, d(x, y))}\left(\frac{d(x, y)}{\phi_{c}^{-1}(t)}\right)^{d_{2}} \exp \left(-\frac{a d(x, y)^{1 / N}}{\phi_{c}^{-1}(t)^{1 / N}}\right) \\
& \leq \frac{c t}{V(x, d(x, y)) \phi_{j}(d(x, y))}+\frac{c_{2}}{V(x, d(x, y))} \exp \left(-\frac{a d(x, y)^{1 / N}}{2}\right)
\end{aligned}
$$

where in the second inequality we used VD and the last inequality follows from the fact that

$$
r^{d_{2}} \leq c_{3} e^{a r^{1 / N} / 2}, \quad r \geq c_{4}>0 .
$$


Without loss of generality, we may and do assume that $N \geq\left(\beta_{2, \phi_{c}}-1\right) / \beta_{1, \phi_{c}}$. In particular, $r^{*}(t) \geq r_{*}(t)$ by the upper bond for $r_{*}(t)$ mentioned at the end of step (ii) and also by choosing $C_{3}>0$ large enough in the definition of $r^{*}(t)$ if necessary.

Next, suppose that there is a constant $C_{3}>0$ in the definition of $r^{*}(t)$ such that

$$
\exp \left(\frac{a}{2} \frac{r^{1 / N}}{\phi_{c}^{-1}(t)^{1 / N}}\right) \geq \frac{c_{5} \phi_{j}(r)}{t}, \quad r \geq r^{*}(t)
$$

holds for some $c_{5}>0$. Then, by (3.7) we have for all $x, y \in M_{0}$ and $t \geq 1$ with $d(x, y) \geq r^{*}(t)$,

$$
p(t, x, y) \leq \frac{c_{6} t}{V(x, d(x, y)) \phi_{j}(d(x, y))} .
$$

Hence, by (1.10) and Lemma 2.3 , for all $x \in M_{0}, t \geq 1$ and $r \geq r^{*}(t)$, we obtain

$$
\begin{aligned}
\int_{B(x, r)^{c}} p(t, x, y) \mu(d y) & \leq c_{6} \int_{B(x, r)^{c}} \frac{t}{V(x, d(x, y)) \phi_{j}(d(x, y))} \mu(d y) \\
& \leq \frac{c_{7} t}{\phi_{j}(r)} \leq c_{8}\left(\frac{\phi_{j}^{-1}(t)}{r}\right)^{\beta_{1, \phi_{j}}}
\end{aligned}
$$

proving (3.3).

We now check (3.8) following the argument of the lower bound of $r_{*}(t)$ in the end of step (ii). First, note from (1.10) that

$$
\frac{c_{5} \phi_{j}(r)}{t} \leq c_{9}\left(\frac{r}{\phi_{j}^{-1}(t)}\right)^{\beta_{2, \phi_{j}}}
$$

Then, (3.8) is a consequence of the following inequality

$$
\exp \left(\frac{a}{2} \frac{r^{1 / N}}{\phi_{c}^{-1}(t)^{1 / N}}\right) \geq c_{9}\left(\frac{r}{\phi_{j}^{-1}(t)}\right)^{\beta_{2, \phi_{j}}}, \quad r \geq r^{*}(t)
$$

that is,

$$
\frac{a}{2}\left(\frac{r}{\phi_{c}^{-1}(t)}\right)^{1 / N} \geq \log c_{9}+\beta_{2, \phi_{j}} \log \frac{r}{\phi_{c}^{-1}(t)}+\beta_{2, \phi_{j}} \log \frac{\phi_{c}^{-1}(t)}{\phi_{j}^{-1}(t)}, \quad r \geq r^{*}(t) .
$$

From this, we see that we can take a proper constant $C_{3}>0$ such that $(3.8)$ is satisfied.

(iv) Let $r \in\left[r_{*}(t), r^{*}(t)\right]$. For any $x \in M_{0}, t \geq 1$ and $r \in\left[r_{*}(t), r^{*}(t)\right]$, we find by the conclusion in step (ii) that

$$
\begin{aligned}
\int_{B(x, r)^{c}} p(t, x, y) \mu(d y) & \leq \int_{B\left(x, r_{*}(t)\right)^{c}} p(t, x, y) \mu(d y) \\
& \leq c_{1}\left(\frac{\phi_{j}^{-1}(t)}{r_{*}(t)}\right)^{\beta_{1, \phi_{j}} / 2}+c_{1} \exp \left(-c_{2} \frac{r_{*}(t)}{\bar{\phi}_{c}^{-1}\left(t / r_{*}(t)\right)}\right) \\
& =: I_{1}+I_{2} .
\end{aligned}
$$


On the one hand, we find by the facts $r_{*}(t) \geq C_{1} \phi_{c}^{-1}(t) \log ^{\left(\beta_{1, \phi_{c}}-1\right) / \beta_{2, \phi_{c}}}\left(\phi_{c}^{-1}(t) / \phi_{j}^{-1}(t)\right)$ and $\phi_{j}^{-1}(t) \leq \phi_{c}^{-1}(t)$ that

$$
\begin{aligned}
I_{1} & \leq c_{3}\left(\frac{\phi_{j}^{-1}(t)}{\phi_{c}^{-1}(t) \log \left(\beta_{1, \phi_{c}}-1\right) / \beta_{2, \phi_{c}}\left(\phi_{c}^{-1}(t) / \phi_{j}^{-1}(t)\right)}\right)^{\beta_{1, \phi_{j}} / 2} \\
& \leq c_{3}\left(\frac{\phi_{j}^{-1}(t)}{\phi_{c}^{-1}(t) \log ^{N}\left(\phi_{c}^{-1}(t) / \phi_{j}^{-1}(t)\right)}\right)^{\left(\beta_{1, \phi_{c}}-1\right) \beta_{1, \phi_{j}} /\left(2 N \beta_{2, \phi_{c}}\right)} \\
& \leq c_{4}\left(\frac{\phi_{j}^{-1}(t)}{r}\right)^{\left(\beta_{1, \phi_{c}}-1\right) \beta_{1, \phi_{j}} /\left(2 N \beta_{2, \phi_{c}}\right)} .
\end{aligned}
$$

On the other hand, without loss of generality we may and do assume that $c_{2} \in(0,1)$ in the term $I_{2}$. By (3.5) and the fact that we can assume in (3.5) that $\frac{2 c_{14}}{\beta_{1, \phi_{j}}} \geq 1$, there is a constant $\theta \in(0,1)$ such that

$$
\begin{aligned}
I_{2} & \leq c_{5}\left(\frac{\phi_{j}^{-1}(t)}{r_{*}(t)}\right)^{\theta} \leq c_{6}\left(\frac{\phi_{j}^{-1}(t)}{\phi_{c}^{-1}(t) \log \left(\beta_{1, \phi_{c}}-1\right) / \beta_{2, \phi_{c}}\left(\phi_{c}^{-1}(t) / \phi_{j}^{-1}(t)\right)}\right)^{\theta} \\
& \leq c_{7}\left(\frac{\phi_{j}^{-1}(t)}{\phi_{c}^{-1}(t) \log ^{N}\left(\phi_{c}^{-1}(t) / \phi_{j}^{-1}(t)\right)}\right)^{\theta\left(\beta_{1, \phi_{c}}-1\right) /\left(N \beta_{2, \phi_{c}}\right)} \leq c_{8}\left(\frac{\phi_{j}^{-1}(t)}{r}\right)^{\theta\left(\beta_{1, \phi_{c}}-1\right) /\left(N \beta_{2, \phi_{c}}\right)} .
\end{aligned}
$$

Combining all the estimates above, we can obtain (3.3) in this case with

$$
\eta=\min \left\{\left(\beta_{1, \phi_{c}}-1\right) \beta_{1, \phi_{j}} /\left(2 N \beta_{2, \phi_{c}}\right), \theta\left(\beta_{1, \phi_{c}}-1\right) /\left(N \beta_{2, \phi_{c}}\right)\right\} .
$$

The proof is complete.

We remark that Lemma 3.6 can quickly lead to the following estimate.

Proposition 3.8. Under VD and (1.10), if $\operatorname{UHKD}(\phi), \mathrm{J}_{\phi, \leq}$ and $\mathrm{E}_{\phi}$ hold, then there is a constant $c>0$ such that for any $t>0$ and $x, y \in M_{0}$,

$$
p(t, x, y) \leq c\left(\frac{1}{V\left(x, \phi^{-1}(t)\right)} \wedge \frac{t}{V(x, d(x, y)) \phi(d(x, y))}\right) .
$$

Proof. According to $\operatorname{UHKD}(\phi)$, we only need to verify that there is a constant $c>0$ such that for any $t>0$ and $x, y \in M_{0}$,

$$
p(t, x, y) \leq \frac{c t}{V(x, d(x, y)) \phi(d(x, y))} .
$$

Following the proof of [CKW4, Lemma 4.7], we can find that, under the assumptions of this proposition, there exist constants $a, c_{0}>0$ and $N \in \mathbb{N}$ such that for all $x, y \in M_{0}$ and $t>0$,

$$
p(t, x, y) \leq \frac{c_{0} t}{V(x, d(x, y)) \phi(d(x, y))}+\frac{c_{0}}{V\left(x, \phi^{-1}(t)\right)} \exp \left(-\frac{a d(x, y)^{1 / N}}{\phi^{-1}(t)^{1 / N}}\right) .
$$


When $d(x, y) \geq \phi^{-1}(t)$, we have by $(3.2)$ with $k=1$ that

$$
\frac{1}{V\left(x, \phi^{-1}(t)\right)} \exp \left(-\frac{a d(x, y)^{1 / N}}{\phi^{-1}(t)^{1 / N}}\right) \leq \frac{c_{1} t}{V(x, d(x, y)) \phi(d(x, y))}
$$

while for $d(x, y) \leq \phi^{-1}(t)$,

$$
\frac{1}{V\left(x, \phi^{-1}(t)\right)} \exp \left(-\frac{a d(x, y)^{1 / N}}{\phi^{-1}(t)^{1 / N}}\right) \leq \frac{c_{2}}{V\left(x, \phi^{-1}(t)\right)} \leq \frac{c_{3} t}{V(x, d(x, y)) \phi(d(x, y))} .
$$

These together with 3.10 yield the estimate 3.9 .

Obviously, the upper bound estimate (3.9) is not as sharp as $\operatorname{UHK}\left(\phi_{j}, \phi_{c}\right)$. However, (3.9) plays a role in the characterizations of parabolic Harnack inequalities below.

Proof of Theorem 1.12. The implications of $(1) \Longrightarrow(4)$ and $(4) \Longrightarrow(5)$ of Theorem 1.12 follow from Propositions $2.1,2.2,2.4$ and 2.5, while the $(5) \Longrightarrow(3)$ part and the $(3) \Longrightarrow(2)$ part follow from Propositions 3.1 and 3.2. Note that by the proof of [CKW1, Lemma 4.21], under VD and $(1.10), \mathrm{E}_{\phi}$ and $\mathrm{J}_{\phi, \leq}$ imply the conservativeness of $(\mathcal{E}, \mathcal{F})$. This together with Proposition 3.5 gives the implication $(2) \Longrightarrow(1)$. This completes the proof of the theorem.

\section{Characterizations of $\mathrm{HK}_{-}\left(\phi_{j}, \phi_{c}\right)$ and $\operatorname{HK}\left(\phi_{j}, \phi_{c}\right)$}

This section is devoted to the proof of Theorem 1.11. Since the proof is similar to that of [CKW4, Theorem 1.14], we only present the different points.

\section{1 $\mathrm{PI}(\phi)+\mathrm{J}_{\phi_{j}}+\mathrm{CSJ}(\phi) \Longrightarrow \mathrm{HK}_{-}\left(\phi_{j}, \phi_{c}\right)$}

Proposition 4.1. If $\mathrm{VD}, \mathrm{RVD}, 1.10, \mathrm{PI}(\phi), \mathrm{J}_{\phi_{j}, \leq}$ and $\mathrm{CSJ}(\phi)$ hold, then we have $\mathrm{NDL}(\phi)$.

Proof. By the proof of [CKW2, Propisition 4.13], under VD, RVD and (1.10,

$$
\operatorname{PI}(\phi)+\mathrm{J}_{\phi, \leq}+\operatorname{CSJ}(\phi) \Longrightarrow \operatorname{EHR} .
$$

Furthermore, by Theorem 1.12, under VD, RVD and (1.10),

$$
\mathrm{PI}(\phi)+\mathrm{J}_{\phi, \leq}+\mathrm{CS}(\phi) \Longrightarrow \mathrm{E}_{\phi},
$$

where we used the fact that $\operatorname{PI}(\phi)$ implies $\operatorname{FK}(\phi)$ under RVD from Proposition 2.1. With those two conclusions at hand, the desired assertion follows from the proof of [CKW2, Proposition 4.9].

The following statement proves that the $(5) \Longrightarrow(1)$ part in Theorem 1.11

Proposition 4.2. If VD, RVD, 1.10, $\mathrm{PI}(\phi), \mathrm{J}_{\phi_{j}}$ and $\operatorname{CSJ}(\phi)$ hold, then we have $\mathrm{HK}_{-}\left(\phi_{j}, \phi_{c}\right)$. 
Proof. By Proposition 4.1, we have NDL $(\phi)$. In particular, for any $x, y \in M_{0}$ and $t>0$ with $d(x, y) \leq c_{0} \phi^{-1}(t)$ for some constant $c_{0}>0$,

$$
p(t, x, y) \geq \frac{c_{1}}{V\left(x, \phi^{-1}(t)\right)} .
$$

Following the arguments in step (ii) for the proof of [CKW1, Proposition 5.4], we know that for all $x, y \in M_{0}$ and $t>0$ with $d(x, y) \geq c_{0} \phi^{-1}(t)$,

$$
p(t, x, y) \geq \frac{c_{2} t}{V(x, d(x, y)) \phi_{j}(d(x, y))} .
$$

Note that, by Theorem 1.12, under assumptions of this proposition, $\operatorname{UHK}\left(\phi_{j}, \phi_{c}\right)$ holds. Combining with all the conclusions, we can obtain the desired assertion.

\subsection{From $\mathrm{HK}_{-}\left(\phi_{j}, \phi_{c}\right)$ to $\mathrm{HK}\left(\phi_{j}, \phi_{c}\right)$}

Proposition 4.3. If $(M, d, \mu)$ is connected and satisfies the chain condition, then $\mathrm{HK}_{-}\left(\phi_{j}, \phi_{c}\right)$ implies $\operatorname{HK}\left(\phi_{j}, \phi_{c}\right)$. In particular, if $\mathrm{VD}, \operatorname{RVD},(1.10), \mathrm{PI}(\phi), \mathrm{J}_{\phi_{j}}$ and $\operatorname{CSJ}(\phi)$ hold, then so does $\operatorname{HK}\left(\phi_{j}, \phi_{c}\right)$.

Proof. We only give a proof for the case of $\beta_{*} \leq 1$. The case of $\beta_{*}>1$ can be proved in a similar way. By the definition of $\mathrm{HK}_{-}\left(\phi_{j}, \phi_{c}\right)$ and Remark 1.16(ii), we only need to establish the lower bound of $\operatorname{HK}\left(\phi_{j}, \phi_{c}\right)$ in the case of $\beta^{*}>1$ and $\phi_{c}(r)$ is not comparable to $\phi_{j}(r)$ on $[1, \infty)$ for $t \in[1, \infty)$ and $x, y \in M_{0}$ with $d(x, y) \geq c_{0} \phi_{c}^{-1}(t)$ for some constant $c_{0}>0$. In this case, a more explicit expression for the lower bound estimate of $\operatorname{HK}\left(\phi_{j}, \phi_{c}\right)$ is given in Remark 1.17(iii) and we only need to establish the exponential part. This can be done by using the standard chaining argument (see the proof of [BGK, Proposition 5.2(i)] or that of [CKW4, Proposition 5.6]). We omit the details here. The second assertion follows from the first one and Theorem 1.11

Proof of Theorem 1.11 By Proposition 2.2, (1) $\Longrightarrow(2)$. (2) $\Longrightarrow(3)$ follows from the remark after Definition 1.10, According to CKW2, Proposition 3.5], under VD and (1.10), NDL $(\phi)$ implies $\operatorname{PI}(\phi)$, and so $\mathrm{FK}(\phi)$ under RVD by Proposition 2.1; while $\operatorname{NDL}(\phi)$ also implies $\mathrm{E}_{\phi}$ thanks to RVD again. With these at hand, by Theorem 1.12 we have $(3) \Longrightarrow(5)$. (5) $\Longrightarrow(6)$ can be seen by Proposition 2.5. Furthermore, by Proposition 4.2 , (6) $\Longrightarrow(1)$. The equivalence between (4) and (6) follows from Propositions 2.1 and 3.2, and the $(2) \Longleftrightarrow(4)$ part of Theorem 1.12. When the space $(M, d, \mu)$ is connected and satisfies the chain condition, $(5) \Longrightarrow(7)$ has been proven in Proposition 4.3 . Clearly, $(7) \Longrightarrow(1)$. The proof is complete.

\section{Applications}

\subsection{Parabolic Harnack inequalities}

In this subsection, we first present a proof for Theorem 1.15 on the stable characterization of parabolic Harnack inequalities. 
Proof of Theorem 1.15. In view of Proposition 3.8, the first assertion of Theorem 1.15 can be established by the same the arguments in [CKW2, Subsection 4.3]. See also [CKW4, Section $6]$ for more details. The second one is a direct consequence of the first one and Theorem 1.11. Indeed, similar to [CKW4, Section 6], one can also derive several characterizations for $\mathrm{PHI}(\phi)$ in the present setting. The details are omitted here. We emphasize that, different from the pure non-local setting as studied in [CKW2], when $J(x, y)$ has lighter tails at infinity, $\operatorname{PHI}(\phi)$ only implies $\mathrm{J}_{\phi, \leq}$ but not $\mathrm{J}_{\phi_{j}, \leq}$ in general. See the example below.

Example 5.1. $\left(\operatorname{PHI}(\phi)\right.$ alone does not imply $\left.\mathrm{J}_{\phi_{j}, \leq}\right)$ Let $M=\mathbb{R}^{d}$, and

$$
J(x, y) \asymp \begin{cases}\frac{1}{|x-y|^{d+\alpha}}, & |x-y| \leq 1 \\ \frac{1}{|x-y|^{d+\beta}}, & |x-y| \geq 1\end{cases}
$$

where $\alpha \in(0,2)$ and $\beta \in(2, \infty)$. We consider the following regular Dirichlet form

$$
\mathcal{E}(f, g)=\iint_{\mathbb{R}^{d} \times \mathbb{R}^{d}}(f(x)-f(y))(g(x)-g(y)) J(x, y) d x d y
$$

and $\mathcal{F}={\overline{C_{1}\left(\mathbb{R}^{d}\right)}}^{\mathcal{E}_{1}}$. It has been proven in [BKKL1, Theorem 3.10] that $\operatorname{PHI}(\phi)$ holds with $\phi(r)=r^{\alpha} \vee r^{2}$. Therefore, for this example $\operatorname{PHI}(\phi)$ holds for any $\beta \in(2, \infty)$; that is, $\operatorname{PHI}(\phi)$ alone does not imply upper bounds of the jumping kernel.

\subsection{Transferring method for heat kernel estimates and parabolic Harnack inequalities}

By the stability results in Theorems 1.11, 1.12 and 1.15, we can obtain heat kernel estimates and parabolic Harnack inequalities for a large class of symmetric jump processes using "transferring method". Namely, we first establish heat kernel estimates and parabolic Harnack inequalities for a jump process with a particular jumping kernel $J(x, y)$, and then use Theorems 1.11, 1.12 and 1.15 to obtain heat kernel estimates and parabolic Harnack inequalities for other symmetric jump processes whose jumping kernels are comparable to $J(x, y)$. For instance, Claim 2 in Example 1.1 gives heat kernel estimates (hence parabolic Harnack inequalities as well) for the subordinate process $Y$. Thus, by Theorems 1.11 and 1.15 , we can obtain the heat kernel estimates (and the parabolic Harnack inequalities) for pure jump processes whose jumping kernels enjoy (1.2). In [CKW1, Section 6.1] and [CKW2, Section 5], we have other examples on fractals that support anomalous diffusions with two-sided heat kernel estimates. The subordination of these diffusion processes enjoy $\operatorname{HK}(\phi)$ and hence $\operatorname{PHI}(\phi)$, which can be served as the base examples. See also [CKW4, Section 7] for related discussions about diffusions with jumps.

\subsection{Examples}

To illustrate the main results of this paper, we present two more examples in this subsection. we will mainly concentrate on the case of

$$
\phi(r)=\phi_{j}(r) \mathbf{1}_{[0,1]}+\phi_{c}(r) \mathbf{1}_{(1, \infty)} .
$$

This corresponds to the cases of $\beta_{*} \leq 1$ and $\beta^{*}>1$, and of $\beta_{*}>1$ with $\phi_{c}(r)=\phi_{j}(r)$ on $[0,1]$ and $\beta^{*}>1$. One can use the similar ideas to give examples in other cases. 
The first example shows that for our main results, the scale function $\phi_{c}(r)$ does not need to be the scaling function corresponding to diffusion processes (e.g. see Example 1.1) even in the case of the Euclidean space.

Example 5.2. Let $M=\mathbb{R}^{d}$ and $\mu(d x)=d x$. Consider the following jumping kernel

$$
J(x, y) \simeq \frac{1}{|x-y|^{d} \phi_{j}(|x-y|)},
$$

where $\phi_{j}$ is a strictly increasing continuous function with $\phi_{j}(0)=0$ and $\phi_{j}(1)=1$ so that

(i) there are constants $c_{1, \phi_{j}}, c_{2, \phi_{j}}>0$ and $0<\beta_{1, \phi_{j}} \leq \beta_{2, \phi_{j}}<2$ such that

$$
c_{1, \phi_{j}}\left(\frac{R}{r}\right)^{\beta_{1, \phi_{j}}} \leq \frac{\phi_{j}(R)}{\phi_{j}(r)} \leq c_{2, \phi_{j}}\left(\frac{R}{r}\right)^{\beta_{2, \phi_{j}}} \text { for all } 0<r \leq R \leq 1
$$

(ii) there are constants $c_{1, \phi_{j}}^{*}, c_{2, \phi_{j}}^{*}>0$ and $1<\beta_{1, \phi_{j}}^{*} \leq \beta_{2, \phi_{j}}^{*}<\infty$ such that

$$
c_{1, \phi_{j}}^{*}\left(\frac{R}{r}\right)^{\beta_{1, \phi_{j}}^{*}} \leq \frac{\phi_{j}(R)}{\phi_{j}(r)} \leq c_{2, \phi_{j}}^{*}\left(\frac{R}{r}\right)^{\beta_{2, \phi_{j}}^{*}} \quad \text { for all } 1 \leq r \leq R .
$$

Let $(\mathcal{E}, \mathcal{F})$ be the associated pure jump Dirichlet form with jumping kernel $J(x, y)$ as above on $L^{2}\left(\mathbb{R}^{d} ; d x\right)$, where $\mathcal{F}=\left\{f \in L^{2}\left(\mathbb{R}^{d} ; d x\right): \mathcal{E}(f, f)<\infty\right\}$. It is easy to check (see [CKK2, p. $1969])$ that $(\mathcal{E}, \mathcal{F})$ is a regular Dirichlet form on $L^{2}\left(\mathbb{R}^{d} ; d x\right)$. By (i), for every $r \in(0,1]$,

$$
\begin{aligned}
\int_{0}^{r} \frac{s}{\phi_{j}(s)} d s & =\sum_{n=1}^{\infty} \int_{2^{-n} r}^{2^{-n+1} r} \frac{s}{\phi_{j}(s)} d s \leq \sum_{n=1}^{\infty} \frac{2^{-n+1} r}{\phi_{j}\left(2^{-n} r\right)} 2^{-n} r \\
& \leq 2 c_{2, \phi_{j}} \frac{r^{2}}{\phi_{j}(r)} \sum_{n=1}^{\infty} 2^{-n\left(2-\beta_{2, \phi_{j}}\right)} \leq \frac{c_{3} r^{2}}{\phi_{j}(r)} .
\end{aligned}
$$

On the other hand,

$$
\int_{0}^{r} \frac{s}{\phi_{j}(s)} d s \geq \frac{1}{\phi_{j}(r)} \int_{0}^{r} s d s=\frac{r^{2}}{2 \phi_{j}(r)} \quad \text { for every } r>0 .
$$

Define

$$
\Phi(r)=\frac{r^{2}}{2 \int_{0}^{r} s / \phi_{j}(s) d s}, \quad r>0
$$

Since

$$
\frac{d}{d r} \Phi(r)=\frac{2 r\left(2 \int_{0}^{r} s / \phi_{j}(s) d s-r^{2} / \phi_{j}(r)\right)}{4\left(\int_{0}^{r} s / \phi_{j}(s) d s\right)^{2}}>0,
$$

$\Phi(r)$ is strictly increasing on $\mathbb{R}_{+}$. Since $\frac{r^{2}}{\Phi(r)}=2 \int_{0}^{r} s / \phi_{j}(s) d s$ is an increasing function,

$$
\frac{\Phi(R)}{\Phi(r)} \leq\left(\frac{R}{r}\right)^{2} \quad \text { for all } R \geq r>0 .
$$

Moreover, by (5.3) and (5.4), $\Phi(r) \leq \phi_{j}(r)$ on $[0, \infty)$, and $\Phi(r)$ is comparable to $\phi_{j}(r)$ on $[0,1]$. 
Define

$$
\phi_{c}(r)= \begin{cases}r^{2}, & r \in(0,1], \\ \frac{\Phi(r)}{\Phi(1)}, & r \in[1, \infty) .\end{cases}
$$

By condition (i), $\phi_{j}(r) \geq c_{2, \phi_{j}}^{-1} r^{\beta_{2, \phi_{j}}}$ for all $r \in(0,1]$ with $\beta_{2, \phi_{j}} \in(0,2)$. So there is a constant $c_{0}>0$ so that $\phi_{c} \leq c_{0} \phi_{j}$ on $[0, \infty)$. Clearly, $\phi_{c}$ is a strictly increasing and continuous function on $\mathbb{R}_{+}$. We claim that there are constants $c_{1, \phi_{c}}>0$ and $\beta_{1, \phi_{c}} \in(1,2]$ such that

$$
c_{1, \phi_{c}}\left(\frac{R}{r}\right)^{\beta_{1, \phi_{c}}} \leq \frac{\phi_{c}(R)}{\phi_{c}(r)} \leq\left(\frac{R}{r}\right)^{2} \text { for all } R \geq r>0 .
$$

Note that the upper bound holds by (5.5), and the lower bounds holds for $0<r \leq R \leq 1$ as $\phi_{c}(r)=r^{2}$ on $[0,1]$. Below, without loss of generality we assume $\beta_{1, \phi_{j}}^{*}<2$ (otherwise, we replace $\beta_{1, \phi_{j}}^{*}$ with $\beta_{1, \phi_{j}}^{*}:=2-\varepsilon$ for small $\varepsilon \in(0,1)$, and (ii) still holds). For any $n \geq 1$ and $r \geq 1$, by a change of variable and (ii),

$$
\begin{aligned}
\int_{0}^{2^{n} r} \frac{s}{\phi_{j}(s)} d s & =\int_{0}^{r} \frac{s}{\phi_{j}(s)} d s+\sum_{k=1}^{n} \int_{2^{k-1} r}^{2^{k} r} \frac{s}{\phi_{j}(s)} d s \\
& \leq \int_{0}^{r} \frac{s}{\phi_{j}(s)} d s+\sum_{k=1}^{n} 2^{2(k-1)} \int_{r}^{2 r} \frac{s}{\phi_{j}\left(2^{k-1} s\right)} d s \\
& \leq \int_{0}^{r} \frac{s}{\phi_{j}(s)} d s+\frac{1}{c_{1, \phi_{j}}^{*}} \sum_{k=1}^{n} 2^{(k-1)\left(2-\beta_{1, \phi_{j}}^{*}\right)} \int_{r}^{2 r} \frac{s}{\phi_{j}(s)} d s \\
& \leq\left(1 \vee \frac{1}{c_{1, \phi_{j}}^{*}}\right) \sum_{k=0}^{n} 2^{(k-1)\left(2-\beta_{1, \phi_{j}}^{*}\right)} \int_{r}^{2 r} \frac{s}{\phi_{j}(r)} d s \\
& \leq c_{4} 2^{n\left(2-\beta_{1, \phi_{j}}^{*}\right)} \int_{0}^{r} \frac{s}{\phi_{j}(s)} d s
\end{aligned}
$$

(note that $\beta_{1, \phi_{j}}^{*}<2$ is used in the last inequality), and so

$$
\phi_{c}\left(2^{n} r\right)=\frac{2^{2 n} r^{2}}{2 \Phi(1) \int_{0}^{2^{n} r} s / \phi_{j}(s) d s} \geq c_{5} 2^{n \beta_{1, \phi_{j}}^{*}} \phi_{c}(r) .
$$

Here the constants $c_{4}, c_{5}>0$ are independent of $r, R$ and $n$. So for any $R>r \geq 1$,

$$
\phi_{c}(R) \geq \phi_{c}\left(2^{\left[\log _{2}(R / r)\right]} r\right) \geq c_{5} 2^{\left[\log _{2}(R / r)\right] \beta_{1, \phi_{j}}^{*}} \phi_{c}(r) \geq c_{6}\left(\frac{R}{r}\right)^{\beta_{1, \phi_{j}}^{*}} \phi_{c}(r) .
$$

For $0<r \leq 1 \leq R$, we have by above and that $\phi_{c}(r)=r^{2}$ on $[0,1]$,

$$
\frac{\phi_{c}(R)}{\phi_{c}(r)}=\frac{\phi_{c}(R)}{\phi_{c}(1)} \frac{\phi_{c}(1)}{r^{2}} \geq c_{6} \frac{R^{\beta_{1, \phi_{j}}^{*}}}{r^{2}} \geq c_{6}\left(\frac{R}{r}\right)^{\beta_{1, \phi_{j}}^{*}} .
$$

The claim (5.6) is proved.

As noted earlier, $\Phi(r)$ and $\phi_{j}(r)$ are comparable on $[0,1]$. Consequently,

$$
\phi(r):=\mathbf{1}_{[0,1]}(r) \phi_{j}(r)+\mathbf{1}_{(1, \infty)}(r) \phi_{c}(r)
$$


is comparable to $\Phi(r)$ on $[0, \infty)$. Hence $\operatorname{PI}(\phi)$ holds by [BKKL1, Proposition 3.2]. On the other hand, by [BKKL1, Lemma 3.9], $\mathrm{EP}_{\phi, \leq}$ is satisfied. So CSJ $(\phi)$ holds by the proofs of Propositions 2.4 and 2.5. Therefore, $\operatorname{HK}\left(\phi_{j}, \phi_{c}\right)$ holds for $(\mathcal{E}, \mathcal{F})$ by Theorem 1.11. This assertion improves [BKKL1, Theorem 1.4 and Corollary 1.5], where an extra condition that $\beta_{1, \phi_{j}}>1$ is required.

We also note that in this example, $\phi_{c}(r)$ does not need to be comparable to the quadratic function $r \mapsto r^{2}$ for all $r>0$. Nevertheless, we can treat the case that pure jump Dirichlet forms with the growth order of $\phi_{j}(r)$ not necessarily strictly less than 2, as in [BKKL1]. By [BGT, Corollaries 2.6.2 and 2.6.4], $\phi_{c}(r)$ and $\phi_{j}(r)$ are comparable on $[1, \infty)$ if and only if $\beta_{2, \beta_{j}}^{*}<2$, in which case, the heat kernel estimate $\operatorname{HK}\left(\phi_{j}, \phi_{c}\right)$ is reduced to $\operatorname{HK}\left(\phi_{j}\right)$ in CKW1] (see Remark 1.16(ii)). Therefore, the "diffusive scaling" appears in $\operatorname{HK}\left(\phi_{j}, \phi_{c}\right)$ only when $\beta_{2, \phi_{j}}^{*} \geq 2$. For example, when $\phi_{j}(r)=r^{\alpha} \vee r^{2}$ for all $r>0$ with $\alpha \in(0,2)$, we can take

$$
\phi_{c}(r):=r^{2} \mathbf{1}_{\{0 \leq r \leq 1\}}+\frac{r^{2}}{\log (1+r)} \mathbf{1}_{\{r>1\}}
$$

and so

$$
\phi(r)=r^{\alpha} \mathbf{1}_{\{0 \leq r \leq 1\}}+\frac{r^{2}}{\log (1+r)} \mathbf{1}_{\{r>1\}} .
$$

It holds that for any $t \geq 1$ and $x, y \in \mathbb{R}^{d}$,

$$
p^{(c)}(t, x, y) \asymp \frac{1}{V\left(x,(t \log (1+t))^{1 / 2}\right)} \exp \left(-\frac{d(x, y)^{2}}{t \log (1+t / d(x, y))}\right) .
$$

This does not belong to the so-called (sub)-Gaussian estimates. Indeed long time behavior of the process is super-diffusive, and its heat kernel estimates are "super-Gaussian".

Our second example is to illustrate our stable characterization of parabolic Harnack inequalities. The assertion of the example below is a counterpart of [CKK2, Theorem 1.4], which only concerns a local version of parabolic Harnack inequalities. One can use the assertion below to study parabolic Harnack inequalities directly for a large class of symmetric jump processes in CKK3.

Example 5.3. Let $M=\mathbb{R}^{d}$ and $\mu(d x)=d x$. Consider the non-local (regular) Dirichlet form $(\mathcal{E}, \mathcal{F})$ on $L^{2}\left(\mathbb{R}^{d} ; d x\right)$ given by

$$
\begin{aligned}
\mathcal{E}(f, f) & =\int_{\mathbb{R}^{d} \times \mathbb{R}^{d}}(f(x)-f(y))^{2} J(x, y) d x d y, \\
\mathcal{F} & =\left\{f \in L^{2}\left(\mathbb{R}^{d} ; d x\right): \mathcal{E}(f, f)<\infty\right\}
\end{aligned}
$$

where $J(x, y)$ is a non-negative symmetric function on $\mathbb{R}^{d} \times \mathbb{R}^{d}$ such that

$$
\begin{aligned}
& J(x, y) \simeq \frac{1}{|x-y|^{d} \phi_{j}(|x-y|)}, \quad|x-y| \leq 1, \\
& J(x, y) \preceq \frac{1}{|x-y|^{d+2}}, \quad|x-y| \geq 1
\end{aligned}
$$

and

$$
\sup _{x \in \mathbb{R}^{d}} \int_{\{|x-y| \geq 1\}}|x-y|^{2} J(x, y) d y<\infty .
$$


Here, $\phi_{j}$ is a strictly increasing continuous function with $\phi_{j}(0)=0$ and $\phi_{j}(1)=1$ so that 5.1 holds with $0<\beta_{1, \phi_{j}} \leq \beta_{2, \phi_{j}}<2$.

We claim that $\mathrm{PHI}(\phi)$ holds with $\phi(r)=\phi_{j}(r) \mathbf{1}_{\{0 \leq r \leq 1\}}+r^{2} \mathbf{1}_{\{r>1\}}$, if and only if UJS holds for the jumping kernel $J(x, y)$ given above. Obviously $\mathrm{J}_{\phi, \leq}$ holds. Next we check the condition $\operatorname{CSJ}(\phi)$. Indeed, for any $x_{0} \in \mathbb{R}^{d}$ and $0<r \leq R$, we choose a Lipschitz continuous function $\varphi: \mathbb{R}^{d} \rightarrow[0,1]$ such that $\varphi(x)=1$ for all $x \in B\left(x_{0}, R\right), \varphi(x)=0$ for all $x \in B\left(x_{0}, R+r\right)^{c}$ and $\|\nabla \varphi\|_{\infty} \leq 2 / r$. Then,

$$
\begin{array}{rlr}
\Gamma(\varphi, \varphi)(x) & =\int_{\mathbb{R}^{d}}(\varphi(x)-\varphi(y))^{2} J(x, y) d y \leq 4 \int_{\mathbb{R}^{d}}\left(\frac{|x-y|^{2}}{r^{2}} \wedge 1\right) J(x, y) d y & \\
& \leq \begin{cases}c_{1} r^{-2} \int_{0}^{r} \frac{s}{\phi_{j}(s)} d s+\int_{r}^{1} \frac{1}{s \phi_{j}(s)} d s+\int_{\{|x-y| \geq 1\}} J(x, y) d y, & 0<r \leq 1 \\
c_{1} r^{-2} \int_{\mathbb{R}^{d}}|x-y|^{2} J(x, y) d y, & r>1 \\
& \leq \frac{c_{2}}{\phi(r)},\end{cases}
\end{array}
$$

where the last inequality is due to (5.3) and (5.7). $\operatorname{CSJ}(\phi)$ is a direct consequence of the above estimate. We now show $\operatorname{PI}(\phi)$ holds. For this, we consider the 1-truncation of the Dirichlet from $(\mathcal{E}, \mathcal{F})$, i.e.

$$
\mathcal{E}^{(1)}(f, f)=\int_{\mathbb{R}^{d} \times \mathbb{R}^{d}}(f(x)-f(y))^{2} J(x, y) \mathbf{1}_{\{|x-y| \leq 1\}} d x d y .
$$

It follows from the proofs of [CKK1, Theorem 3.5 and Proposition 3.8] that NDL $(\phi)$ holds for $\left(\mathcal{E}^{(1)}, \mathcal{F}\right)$. (In fact, the paper [CKK1] only treats the case that $\phi_{j}(r)=r^{\alpha}$ for some $\alpha \in(0,2)$, but the extension to the general $\phi_{j}$ as above is easy.) Then, by [CKW2, Proposition 3.5(1)], $\operatorname{PI}(\phi)$ is satisfied for $\left(\mathcal{E}^{(1)}, \mathcal{F}\right)$. Denote by $\Gamma^{(1)}(f, f)$ and $\Gamma(f, f)$ the carré du-Champ operator for $\left(\mathcal{E}^{(1)}, \mathcal{F}\right)$ and $(\mathcal{E}, \mathcal{F})$, respectively. Since $\Gamma^{(1)}(f, f) \leq \Gamma(f, f)$ for all $f \in \mathcal{F}$, we have $\operatorname{PI}(\phi)$ for $(\mathcal{E}, \mathcal{F})$ too. Therefore, combining all the conclusions above, we have by Theorem 1.15 that $\operatorname{PHI}(\phi)$ holds for $(\mathcal{E}, \mathcal{F})$ if and only if UJS holds for $J(x, y)$.

\section{Appendix: Proof of assertions in Example 1.1}

We begin with the following simple statement.

Lemma 6.1. Let $S$ be the subordinator given in Subsection 1.1. Then,

(i) for all $r>0$,

$$
f(r) \simeq r^{\gamma_{1}} \wedge r
$$

(ii) for any $t>0, S_{t}$ has a density function $\eta_{t}(u)$ with respect to the Lebesgue measure.

(iii) $\mathbb{E} S_{1}<\infty$, and so for all $t>0, \mathbb{E} S_{t}=t \mathbb{E} S_{1}<\infty$.

Proof. (i) It follows from elementary calculations as shown below. For $r>1$, by $\gamma_{2}>\gamma_{1}$,

$$
f(r) \leq \int_{0}^{\infty}\left(1-e^{-r s}\right) \frac{1}{s^{1+\gamma_{1}}} d s \leq c_{1} r^{\gamma_{1}}
$$


where the last inequality follows from the standard $\gamma_{1}$-subordinator whose Lévy measure is comparable with $\frac{1}{s^{1+\gamma_{1}}} d s$. On the other hand, according to the fact that $1-e^{-u} \geq e^{-1} u$ for all $u \in(0,1)$, we have

$$
f(r) \geq \int_{0}^{1 / r}\left(1-e^{-s r}\right) \frac{1}{s^{1+\gamma_{1}}} d s \geq e^{-1} \int_{0}^{1 / r} s r \frac{1}{s^{1+\gamma_{1}}} d s \geq c_{2} r^{\gamma_{1}} .
$$

For $r \in(0,1]$,

$$
\begin{aligned}
f(r) & =\int_{0}^{1}\left(1-e^{-r s}\right) \frac{1}{s^{1+\gamma_{1}}} d s+\int_{1}^{1 / r}\left(1-e^{-r s}\right) \frac{1}{s^{1+\gamma_{2}}} d s+\int_{1 / r}^{\infty}\left(1-e^{-r s}\right) \frac{1}{s^{1+\gamma_{2}}} d s \\
& \leq \int_{0}^{1} r s \frac{1}{s^{1+\gamma_{1}}} d s+\int_{1}^{1 / r} r s \frac{1}{s^{1+\gamma_{2}}} d s+\int_{1 / r}^{\infty} \frac{1}{s^{1+\gamma_{2}}} d s \\
& \leq c_{3} r
\end{aligned}
$$

where in the first inequality we used the fact that $1-e^{-u} \leq u$ for all $u>0$, and the last inequality follows from $\gamma_{2}>1$. On the other hand, for $r \in(0,1]$, we also have

$$
f(r) \geq \int_{0}^{1}\left(1-e^{-r s}\right) \frac{1}{s^{1+\gamma_{1}}} d s \geq e^{-1} r \int_{0}^{1} \frac{1}{s^{1+\gamma_{1}}} d s \geq c_{4} r .
$$

Combining with all the conclusions above, we obtain the first assertion.

(ii) This immediately follows from

$$
\lim _{r \rightarrow \infty} \frac{f(r)}{r^{\gamma_{1}}} \in(0, \infty)
$$

see e.g. [KS, Theorem 1].

(iii) This is a consequence of $\int_{0}^{\infty} s \nu(s) d s=\infty$, due to the fact that $\gamma_{2}>1$. See, e.g., the proof of [BSW, Proposition 1.2].

Proof of Claim 1 in Example 1.1. (i) Let $p(t, x, y)$ be the transition density function of the process $Y$. Let $\eta_{t}(u)$ be the density function of $S_{t}$ as in Lemma 6.1(2). Then,

$$
p(t, x, y)=\int_{0}^{\infty} q(u, x, y) \eta_{t}(u) d u, \quad t>0, x, y \in M,
$$

which yields that the jumping kernel $J(x, y)$ of the process $Y$ is given by

$$
J(x, y)=\int_{0}^{\infty} q(u, x, y) \nu(u) d u
$$

Next, we write

$$
J(x, y)=\int_{0}^{d(x, y) \vee 1} q(u, x, y) \nu(u) d u+\int_{d(x, y) \vee 1}^{\infty} q(u, x, y) \nu(u) d u=: I_{1}+I_{2},
$$

and consider bounds for $J(x, y)$.

First, let $x, y \in M$ with $d(x, y) \leq 1$. Then, by $\gamma_{2}>\gamma_{1}$,

$$
I_{2} \leq \int_{1}^{\infty} q(u, x, y) \nu(u) d u \leq \int_{1}^{\infty} q(u, x, y) \frac{1}{u^{1+\gamma_{1}}} d u .
$$


This along with $I_{1}$ yields that

$$
J(x, y) \leq \int_{0}^{\infty} q(u, x, y) \frac{1}{u^{1+\gamma_{1}}} d u \leq \frac{c_{1}}{V(x, d(x, y)) d(x, y)^{\beta \gamma_{1}}}=\frac{c_{1}}{V(x, d(x, y)) d(x, y)^{\alpha_{1}}},
$$

where the inequality above follows from the standard $\gamma_{1}$ subordination of the process $X$. On the other hand, by changing the variable $s \rightarrow u=d(x, y)^{\beta} / s$, we find that

$$
\begin{aligned}
I_{1} & =d(x, y)^{-\gamma_{1} \beta} \int_{d(x, y)^{\beta}}^{\infty} \frac{1}{V\left(x, d(x, y) / u^{1 / \beta}\right)} \exp \left(-c u^{1 /(\beta-1)}\right) u^{-1+\gamma_{1}} d u \\
& \geq c_{2} d(x, y)^{-\gamma_{1} \beta} \int_{1}^{2} \frac{1}{V\left(x, d(x, y) / u^{1 / \beta}\right)} d u \geq \frac{c_{3}}{V(x, d(x, y)) d(x, y)^{\alpha_{1}}}
\end{aligned}
$$

where the last inequality follows from VD.

Second, let $x, y \in M$ with $d(x, y) \geq 1$. Then, also by $\gamma_{2}>\gamma_{1}$,

$$
\begin{aligned}
I_{1} & =\int_{0}^{1} q(u, x, y) \nu(u) d u+\int_{1}^{d(x, y)} q(u, x, y) \nu(u) d u \\
& \leq \int_{0}^{1} q(u, x, y) u^{-1-\gamma_{2}} d u+\int_{1}^{d(x, y)} q(u, x, y) \nu(u) d u,
\end{aligned}
$$

which along with $I_{2}$ and the change of variable $s \rightarrow u=d(x, y)^{\beta} / s$ yields that

$$
\begin{aligned}
J(x, y) \leq & \int_{0}^{\infty} q(u, x, y) \frac{1}{u^{1+\gamma_{2}}} d u \\
= & d(x, y)^{-\gamma_{2} \beta} \int_{0}^{\infty} \frac{1}{V\left(x, d(x, y) / u^{1 / \beta}\right)} \exp \left(-c u^{1 /(\beta-1)}\right) u^{-1+\gamma_{2}} d u \\
\leq & c_{4} d(x, y)^{-\gamma_{2} \beta} \int_{0}^{1} \frac{1}{V\left(x, d(x, y) / u^{1 / \beta}\right)} d u \\
& +c_{5} d(x, y)^{-\gamma_{2} \beta} \int_{1}^{\infty} \frac{1}{V\left(x, d(x, y) / u^{1 / \beta}\right)} \exp \left(-\frac{c}{2} u^{1 /(\beta-1)}\right) d u \\
\leq & \frac{c_{6}}{V(x, d(x, y)) d(x, y)^{\gamma_{2} \beta}}=\frac{c_{6}}{V(x, d(x, y)) d(x, y)^{\alpha_{2}}},
\end{aligned}
$$

where the last inequality also follows from VD. Similarly, it holds that

$$
\begin{aligned}
I_{2} & =d(x, y)^{-\gamma_{2} \beta} \int_{0}^{d(x, y)^{\beta}} \frac{1}{V\left(x, d(x, y) / u^{1 / \beta}\right)} \exp \left(-c u^{1 /(\beta-1)}\right) u^{-1+\gamma_{2}} d u \\
& \geq c_{7} d(x, y)^{-\gamma_{2} \beta} \int_{1 / 2}^{1} \frac{1}{V\left(x, d(x, y) / u^{1 / \beta}\right)} d u \\
& \geq \frac{c_{8}}{V(x, d(x, y)) d(x, y)^{\gamma_{2} \beta}}=\frac{c_{8}}{V(x, d(x, y)) d(x, y)^{\alpha_{2}}} .
\end{aligned}
$$

This completes the proof of the first assertion.

(ii) For any $x \in M$ and $r>0$,

$$
\int_{B(x, r)^{c}} p(t, x, y) \mu(d y)=\int_{B(x, r)^{c}} \int_{0}^{\infty} q(u, x, y) \eta_{t}(u) d u \mu(d y)
$$




$$
\begin{aligned}
& =\int_{0}^{\infty} \eta_{t}(u) \int_{B(x, r)^{c}} q(u, x, y) \mu(d y) d u \\
& \leq c_{1} \int_{0}^{\infty} \eta_{t}(u) \frac{u}{r^{\beta}} d u=\frac{c_{1}}{r^{\beta}} \mathbb{E} S_{t}=c_{2} \frac{t}{r^{\beta}},
\end{aligned}
$$

where the inequality concerning the tail probability of the diffusion $X$ follows from (1.1), see e.g. [GH], and in the last equality we used Lemma 6.1(3). Therefore, we find that for any $x \in M$ and $r>0$,

$$
\mathbb{P}^{x}\left(\tau_{B(x, r)}^{Y} \leq t\right) \leq c_{3} t / r^{\beta}
$$

where

$$
\tau_{B(x, r)}^{Y}=\inf \left\{t>0: Y_{t} \notin B(x, r)\right\} .
$$

Now let us consider the following jumping kernel:

$$
J^{*}(x, y)= \begin{cases}J(x, y), & d(x, y) \leq 1 \\ \frac{1}{V(x, d(x, y)) d(x, y)^{\alpha_{1}}}, & d(x, y) \geq 1 .\end{cases}
$$

Clearly, $J^{*}(x, y)$ is comparable to the jumping kernel corresponding to the standard $\gamma_{1}$-subordination of the process $X$. Let $Y^{*}:=\left(Y_{t}^{*}\right)_{t \geq 0}$ be the symmetric Markov jump process associated with the jumping kernel $J^{*}(x, y)$, and let $p^{Y^{*}}(t, x, y)$ be the transition density function of the process $Y^{*}$. Since $X$ is conservative, $Y^{*}$ is also conservative. According to the stability result for heat kernel upper bounds in [CKW1, Theorem 1.15], we have

$$
p^{*}(t, x, y) \preceq \frac{1}{V\left(x, t^{1 / \alpha_{1}}\right)} \wedge \frac{t}{V(x, d(x, y)) d(x, y)^{\alpha_{1}}} .
$$

Now, let $Y^{*, 1}$ be the truncated process associated with the process $Y^{*}$ by removing jumps bigger than 1 ; that is, the jumping kernel of the process $Y^{*, 1}$ is given by

$$
J^{*, 1}(x, y)=J(x, y) \mathbf{1}_{\{d(x, y) \leq 1\}} .
$$

By [CKW1, Theorem 1.15 and Lemma 4.19], there are constants $c_{4}>0$ and $c_{5} \in(0,1)$ such that for all $x \in M$ and $t>0$,

$$
\mathbb{P}^{x}\left(\tau_{B(x, r)}^{Y^{*, 1}} \leq t\right) \leq \frac{c_{4} t}{r^{\alpha_{1}}}+c_{5},
$$

which along with [CKW1, Lemma 7.8] (note that $Y^{*, 1}$ is also the truncated process associated with the process $Y$ by removing jumps bigger than 1) yields that for all $x \in M$ and $t, r>0$,

$$
\mathbb{P}^{x}\left(\tau_{B(x, r)}^{Y} \leq t\right) \leq \frac{c_{4} t}{r^{\alpha_{1}}}+c_{5}+c_{6} t
$$

In particular, there are constants $c_{0}, \varepsilon_{0} \in(0,1)$ such that for all $r \leq 1$ and $x \in M$,

$$
\mathbb{P}^{x}\left(\tau_{B(x, r)}^{Y} \leq c_{0} r^{\alpha_{1}}\right) \leq \varepsilon_{0}
$$

Hence, according to (6.1) and (6.2), there are constants $c_{*}, \varepsilon_{*} \in(0,1)$ such that for all $r>0$ and $x \in M$,

$$
\mathbb{P}^{x}\left(\tau_{B(x, r)}^{Y} \leq c_{*}\left(r^{\alpha_{1}} \vee r^{\beta}\right)\right) \leq \varepsilon_{*} .
$$


This immediately yields

$$
\left.\mathbb{E}^{x}\left[\tau_{B(x, r)}^{Y}\right] \geq c_{*}\left(r^{\alpha_{1}} \vee r^{\beta}\right) \mathbb{P}^{x}\left(\tau_{B(x, r)}^{Y} \geq c_{*}\left(r^{\alpha_{1}} \vee r^{\beta}\right)\right) \geq\left(1-\varepsilon_{*}\right) c_{*}\left(r^{\alpha_{1}} \vee r^{\beta}\right)\right)
$$

On the other hand, the estimate above along with (the proofs of) CKW1, Lemma 3.4, Proposition 3.6 and Proposition 2.3(5)] gives us that for any $x_{0} \in M$ and $r>0$,

$$
\operatorname{cap}_{Y}\left(B\left(x_{0}, r\right), B\left(x_{0}, 2 r\right)\right) \leq \begin{cases}c_{7} V\left(x_{0}, r\right) / r^{\alpha_{1}}, & r \leq 1 \\ c_{7} V\left(x_{0}, r\right) / r^{\beta}, & r \geq 1\end{cases}
$$

Note that, in the argument above we need to use the fact that for any $x \in M$ and $r>0$,

$$
\int_{\{y \in M: d(x, y) \geq r\}} J(x, y) \mu(d y) \leq \frac{c_{8}}{r^{\alpha_{1}} \vee r^{\alpha_{2}}} \leq \frac{c_{8}}{r^{\alpha_{1}} \vee r^{\beta}}
$$

Next, we verify the upper bound of $\mathbb{E}^{x} \tau_{B(x, r)}^{Y}$ and the lower bound of the capacity. For $t \geq 1$ and $x, y \in M$,

$$
\begin{aligned}
p(t, x, y) & \leq c_{1} \int_{0}^{\infty} \frac{1}{V\left(x, u^{1 / \beta}\right)} \eta_{t}(u) d u \\
& =c_{1} \int_{0}^{t} \frac{1}{V\left(x, u^{1 / \beta}\right)} \eta_{t}(u) d u+\frac{c_{1}}{V\left(x, t^{1 / \beta}\right)} \int_{t}^{\infty} \eta_{t}(u) d u \\
& \leq \frac{c_{2}}{V\left(x, t^{1 / \beta}\right)} \int_{0}^{t}\left(\frac{t}{u}\right)^{d / \beta} \eta_{t}(u) d u+\frac{c_{1}}{V\left(x, t^{1 / \beta}\right)} \\
& \leq \frac{c_{2} t^{d / \beta}}{V\left(x, t^{1 / \beta}\right)} \int_{0}^{\infty} u^{-d / \beta} \eta_{t}(u) d u+\frac{c_{1}}{V\left(x, t^{1 / \beta}\right)},
\end{aligned}
$$

where the constant $d$ comes from VD. Let $f$ be the Bernstein function of the subordinator $S$. According to GRW, p. 298], we know that for all $t>0$,

$$
\int_{0}^{\infty} u^{-d / \beta} \eta_{t}(u) d u=\int_{0}^{\infty} u^{-d / \beta-1} e^{-t f(u)} d u \leq c_{3} t^{-d / \beta}
$$

where in the inequality we used the fact that $f(r) \asymp r \wedge r^{\gamma_{1}}$ from Lemma 6.1(1) and the Abelian and Tauberian theorem (see [BGT, Theorems 1.7.1 and 1.7.1']). Therefore, for any $x, y \in M$ and $t \geq 1$,

$$
p(t, x, y) \leq \frac{C_{1}}{V\left(x, t^{1 / \beta}\right)} .
$$

Next, we consider the case that $t \leq 1$ and $x, y \in M$,

$$
\begin{aligned}
p(t, x, y) & \leq c_{4} \int_{0}^{\infty} \frac{1}{V\left(x, u^{1 / \beta}\right)} \eta_{t}(u) d u \\
& \leq c_{4} \int_{0}^{t^{1 / \gamma_{1}}} \frac{1}{V\left(x, u^{1 / \beta}\right)} \eta_{t}(u) d u+\frac{c_{4}}{V\left(x, t^{1 /\left(\beta \gamma_{1}\right)}\right)} \int_{t^{1 / \gamma_{1}}}^{\infty} \eta_{t}(u) d u \\
& \leq \frac{c_{5}}{V\left(x, t^{1 /\left(\beta \gamma_{1}\right)}\right)} \int_{0}^{t^{1 / \gamma_{1}}}\left(\frac{t^{1 / \gamma_{1}}}{u}\right)^{d / \beta} \eta_{t}(u) d u+\frac{c_{4}}{V\left(x, t^{1 / \alpha_{1}}\right)}
\end{aligned}
$$




$$
\leq \frac{c_{5} t^{d / \alpha_{1}}}{V\left(x, t^{1 / \alpha_{1}}\right)} \int_{0}^{\infty} u^{-d / \beta} \eta_{t}(u) d u+\frac{c_{4}}{V\left(x, t^{1 / \alpha_{1}}\right)}
$$

Following the same argument as the case $t \geq 1$, we can apply $f(r) \asymp r \wedge r^{\gamma_{1}}$ and the Abelian and Tauberian theorem again, and obtain that

$$
\int_{0}^{\infty} u^{-d / \beta} \eta_{t}(u) d u \simeq t^{-d / \alpha_{1}}, \quad t \in(0,1] .
$$

Hence, for all $t \in(0,1]$ and $x, y \in M$,

$$
p(t, x, y) \leq \frac{C_{2}}{V\left(x, t^{1 / \alpha_{1}}\right)}
$$

Putting both estimates together, we have for all $x, y \in M$ and $t>0$,

$$
p(t, x, y) \leq \frac{C_{0}}{V\left(x, t^{1 / \alpha_{1}} \wedge t^{1 / \beta}\right)} .
$$

This along with CKW1, Propsition 7.3] yields the Faber-Krahn inequality with rate function $r^{\alpha_{1}} \vee r^{\beta}$. Then, by [CKW1, Lemma 4.14], for any $x \in M$ and $r>0$,

$$
\mathbb{E}^{x} \tau_{B(x, r)} \leq c_{5}\left(r^{\alpha_{1}} \vee r^{\beta}\right)
$$

On the other hand, recall that

$$
\operatorname{cap}_{Y}\left(B\left(x_{0}, r\right), B\left(x_{0}, 2 r\right)\right)=\inf \left\{\mathcal{E}_{Y}(\phi, \phi): \phi \text { is a cut-off function for } B\left(x_{0}, r\right) \text { and } B\left(x_{0}, 2 r\right)\right\}
$$

and

$$
\lambda_{\min }\left(B\left(x_{0}, 2 r\right)\right)=\inf \left\{\frac{\mathcal{E}_{Y}(f, f)}{\mu\left(f^{2}\right)}:\left.f\right|_{B\left(x_{0}, 2 r\right)^{c}}=0\right\} .
$$

It holds that

$$
\operatorname{cap}\left(B\left(x_{0}, r\right), B\left(x_{0}, 2 r\right)\right) \geq V\left(x_{0}, r\right) \lambda_{\min }\left(B\left(x_{0}, 2 r\right)\right) .
$$

Therefore, the lower bound for the capacity follows from the Faber-Krahn inequality above and 6.4.

Proof of Claim 2 in Example 1.1. With (6.3) and Claim 1 of Example 1.1 at hand, we know from Theorem 1.12 that $\operatorname{UHK}\left(\phi_{j}, \phi_{c}\right)$ holds for the process $Y$ with $\phi(r)=r^{\alpha_{1}} \vee r^{\beta}$. Furthermore, for any $t \geq 1$ and $x, y \in M$ with $d(x, y) \leq t^{1 / \beta}$, and any $c_{0}>1$, by (1.1), Lemma 6.1(1) and [Mi, Proposition 2.4],

$$
\begin{aligned}
p(t, x, y) & =\int_{0}^{\infty} q(u, x, y) \eta_{t}(u) d u \geq \int_{t}^{c_{0} t} q(u, x, y) \eta_{t}(u) d u \\
& \geq \frac{c_{1}\left(c_{0}\right)}{V\left(x, t^{1 / \beta}\right)} \mathbb{P}\left(t \leq S_{t} \leq c_{0} t\right) \geq \frac{c_{1}\left(c_{0}\right)}{V\left(x, t^{1 / \beta}\right)} .
\end{aligned}
$$

Similarly, for any $t \in(0,1]$ and $x, y \in M$ with $d(x, y) \leq t^{1 / \alpha_{1}}$, and any $c_{0}>1$,

$$
p(t, x, y)=\int_{0}^{\infty} q(u, x, y) \eta_{t}(u) d u \geq \int_{t^{1 / \gamma_{1}}}^{c_{0} t^{1 / \gamma_{1}}} q(u, x, y) \eta_{t}(u) d u
$$




$$
\geq \frac{c_{3}\left(c_{0}\right)}{V\left(x, t^{1 / \alpha_{1}}\right)} \mathbb{P}\left(t^{1 / \gamma_{1}} \leq S_{t} \leq c_{0} t^{1 / \gamma_{1}}\right) \geq \frac{c_{4}\left(c_{0}\right)}{V\left(x, t^{1 / \alpha_{1}}\right)}
$$

where in the second inequality we also used the fact that $\alpha_{1}=\gamma_{1} \beta$. Thus, by these two estimates above, NL $(\phi)$ holds. Therefore, according to Theorem 1.11. HK- $\left(\phi_{j}, \phi_{c}\right)$ holds for the process $Y$, which is exactly (1.3) and (1.4). If in addition $(M, d, \mu)$ is connected and satisfies the chain condition, then $\operatorname{HK}\left(\phi_{j}, \phi_{c}\right)$ holds, which is 1.4$)$. This establishes Claim 2 in Example 1.1 .

Acknowledgements. We thank Professor Alexander Grigor'yan concerning the question about the heat kernel of the subordinate process $Y$ in Example 1.1, which prompted our systematic investigation carried out in this paper. The research of Zhen-Qing Chen is partially supported by Simons Foundation Grant 520542, a Victor Klee Faculty Fellowship at UW, and NNSFC grant 11731009. The research of Takashi Kumagai is supported by JSPS KAKENHI Grant Number JP17H01093 and by the Alexander von Humboldt Foundation. The research of Jian Wang is supported by the National Natural Science Foundation of China (No. 11831014), the Program for Probability and Statistics: Theory and Application (No. IRTL1704) and the Program for Innovative Research Team in Science and Technology in Fujian Province University (IRTSTFJ).

\section{References}

[AB] S. Andres and M.T. Barlow. Energy inequalities for cutoff-functions and some applications. J. Reine Angew. Math. 699 (2015), 183-215.

[BB] M.T. Barlow and R.F. Bass. Stability of parabolic Harnack inequalities. Trans. Amer. Math. Soc. 356 (2003), 1501-1533.

[BBCK] M.T. Barlow, R.F. Bass, Z.-Q. Chen and M. Kassmann. Non-local Dirichlet forms and symmetric jump processes. Trans. Amer. Math. Soc. 361 (2009), 1963-1999.

[BBK1] M.T. Barlow, R.F. Bass and T. Kumagai. Stability of parabolic Harnack inequalities on metric measure spaces. J. Math. Soc. Japan 58 (2006), 485-519.

[BBK2] M.T. Barlow, R. F. Bass and T. Kumagai. Parabolic Harnack inequality and heat kernel estimates for random walks with long range jumps. Math. Z. 261 (2009), 297-320.

[BGK] M.T. Barlow, A. Grigor'yan and T. Kumagai. On the equivalence of parabolic Harnack inequalities and heat kernel estimates. J. Math. Soc. Japan 64 (2012), 1091-1146.

[BKKL1] J. Bae, J. Kang, P. Kim and J. Lee. Heat kernel estimates for symmetric jump processes with mixed polynomial growths. To appear in Ann. Probab., available at arXiv:1804.06918.

[BKKL2] J. Bae, J. Kang, P. Kim and J. Lee. Heat kernel estimates and their stabilities for symmetric jump processes with general mixed polynomial growths on metric measure spaces, arXiv:1904.10189.

[BGT] N.H. Bingham, C.M. Goldie and J.L. Teugels. Regular Variation, Encyclopedia of Mathematics and Its Applications 27, Cambridge Univ. Press, Cambridge, 1987.

[BSW] B. Böttcher, R.L. Schilling and J. Wang. Constructions of coupling processes for Lévy processes and their applications. Stoch. Proc. Appl. 121 (2011), 1201-1216.

[CKK1] Z.-Q. Chen, P. Kim and T. Kumagai. Weighted Poincaré inequality and heat kernel estimates for finite range jump processes. Math. Ann. 342 (2008), 833-883.

[CKK2] Z.-Q. Chen, P. Kim and T. Kumagai. On heat kernel estimates and parabolic Harnack inequality for jump processes on metric measure spaces. Acta Math. Sin. (Engl. Ser.) 25 (2009), 1067-1086. 
[CKK3] Z.-Q. Chen, P. Kim and T. Kumagai. Global heat kernel estimates for symmetric jump processes. Trans. Amer. Math. Soc. 363 (2011), 5021-5055.

[CK] Z.-Q. Chen and T. Kumagai. Heat kernel estimates for stable-like processes on $d$-sets. Stochastic Process Appl. 108 (2003), 27-62.

[CKW1] Z.-Q. Chen, T. Kumagai and J. Wang. Stability of heat kernel estimates for symmetric non-local Dirichlet forms. To appear in Memoirs Amer. Math. Soc., available at arXiv:1604.04035.

[CKW2] Z.-Q. Chen, T. Kumagai and J. Wang. Stability of parabolic Harnack inequalities for symmetric non-local Dirichlet forms. To appear in J. European Math. Soc., available at arXiv:1609.07594.

[CKW3] Z.-Q. Chen, T. Kumagai and J. Wang. Elliptic Harnack inequalities for symmetric non-local Dirichlet forms. J. Math. Pures Appl. 125 (2019), 1-42.

[CKW4] Z.-Q. Chen, T. Kumagai and J. Wang. Heat kernel and parabolic Harnack inequalities for symmetric Dirichlet forms. available at arXiv.

[FOT] M. Fukushima, Y. Oshima and M. Takeda. Dirichlet Forms and Symmetric Markov Processes. de Gruyter, Berlin, 2nd rev. and ext. ed., 2011.

[GRW] M. Gordina, M. Röckner and F.-Y. Wang. Dimension-independent Harnack inequalities for subordinated semigroups. Potential Anal. 34 (2011), 293-307.

[GH] A. Grigor'yan and J. Hu. Upper bounds of heat kernels on doubling spaces. Mosco Math. J. 14 (2014), 505-563.

[GHH] A. Grigor'yan, E. Hu and J. Hu. Two-sided estimates of heat kernels of jump type Dirichlet forms. Advances in Math. 330 (2018), 433-515.

[GT] A. Grigor'yan and A. Telcs. Two-sided estimates of heat kernels on metric measure spaces. Ann. Probab. 40 (2012), 1212-1284.

[KS] V. Knopova and R.L. Schilling. A note on the existence of transition probability densities for Lévy processes. Forum Math. 25 (2013), 125-149.

[Mi] A. Mimica. Heat kernel estimates for subordinate Brownian motions. Proc. Lond. Math. Soc. 113 (2016), 627-648.

\section{Zhen-Qing Chen}

Department of Mathematics, University of Washington, Seattle, WA 98195, USA

School of Mathematics and Statistics, Beijing Institute of Technology, China

E-mail: zqchen@uw.edu

Takashi Kumagai:

Research Institute for Mathematical Sciences, Kyoto University, Kyoto 606-8502, Japan

Email: kumagai@kurims.kyoto-u.ac.jp

\section{Jian Wang:}

College of Mathematics and Informatics \& Fujian Key Laboratory of Mathematical Analysis and Applications (FJKLMAA), Fujian Normal University, 350007, Fuzhou, P.R. China.

Email: jianwang@fjnu.edu.cn 